\title{
Generalized Route Planning Model for Hazardous Material Transportation with VaR and Equity Considerations
}

\author{
Yingying Kang \\ Sabre Airline Solutions, Dallas, TX, USA \\ yingying. kang@sabre.com \\ Rajan Batta and Changhyun Kwon* \\ Department of Industrial \& Systems Engineering, University at Buffalo, the State University \\ of New York, Buffalo, NY, USA \\ batta@buffalo.edu, chkwon@buffalo.edu
}

August 10, 2013

\begin{abstract}
Recently, the Value-at-Risk (VaR) framework was introduced for the routing problem of a single hazmat trip. In this paper, we extend the VaR framework in two important ways. First, we show how to apply the VaR concept to a more realistic multi-trip multi-hazmat type framework, which determines routes that minimize the global VaR value while satisfying equity constraints. Second, we show how to embed the algorithm for the single hazmat trip problem into a Lagrangian relaxation framework to obtain an efficient solution method for this general case. We test our computational experience based on a real-life hazmat routing scenario in the Albany district of New York State. Our results indicate that one can achieve a high degree of risk dispersion while controlling the VaR value within the desired confidence level.
\end{abstract}

Keywords: Value-at-Risk; multi-trip hazmat transportation; social risk mitigation; risk equity; dissimilar path

${ }^{*}$ Corresponding Author 


\section{Introduction}

Hazardous material transportation planning has been well recognized as a Low-Probability-HighConsequence (LPHC) problem. A study of available data on hazmat transportation accident statistics in North America reveals that accident probabilities are extremely small, usually estimated at $10^{-6}$ per mile [18]. However, the low likelihood of hazmat accidents results in an insufficient or inaccurate set of historical records to predict the accident probabilities of future hazmat accidents. On the other hand, due to their disastrous consequences, prediction of accident probabilities of hazmat accidents is critical to reduction of societal risk. To illustrate this point further we note that there were only 167,680 hazmat transportation incidents for the ten year period from 2000 to 2009, which resulted in a total of just 133 fatalities and 2,784 injuries, while damage caused was $\$ 637,270,767$ [39] due to the evacuation and cleanup efforts needed after a hazmat accident. Hazmat transport planning has received the attention from numerous Operations Research and Management Science researchers. Many models have been created, with objectives to minimize the expected risk [4, 20], maximum risk [14], and the mean-variance of the risk [14]. There are some other models that consider balancing hazmat risk and transportation cost [30, 40]. A common characteristic of all of these approaches is that they rely on hazmat accident statistics being available and accurate.

To overcome the drawback associated with the reliance on the availability and the accuracy of hazmat accident statistics, Kang et al. [22] have introduced the Value-at-Risk (VaR) concept as it applies to hazmat routing and risk assessment. VaR provides a flexible decision making tool that covers various risk preferences. By varying a model parameter that represents a certain probability threshold, one can be flexibly risk-averse up to the level of the maximum risk model of Erkut and Ingolfsson [14]. Unlike other models that can be flexibly adjusted such as the mean-variance model, the model parameter is easier to understand; it is a probability threshold.

While Kang et al. [22] focused on a single trip case, we define a trip as a shipment carrying a type of hazmat from an origin to a destination. In this paper, we expand on the work of Kang et al. [22] in two significant directions. First, we demonstrate its application to the multi-trip, multi-hazmat situation while considering equity as a constraint. Second, we use Lagrangian relaxation coupled with the single trip solution to develop an efficient algorithm. By building a set of dissimilar paths among the first $k$ shortest paths of each trip, we reduced the search space for optimal solution 
and significantly reduce the computational effort for large-sized network problem. Subsequently, computational testing is conducted on a realistic routing scenario in Albany, New York. Our principal finding is that by modest increases in overall VaR value one can achieve quite stringent levels of risk equity.

The remainder of this paper is organized as follows. The next section reviews some directly related literature. In Section 3, we present the mathematical formulation of the generalized routing problems for hazmat transport based on the hazmat VaR model. Sections 4 and 5 develop the corresponding solution framework on a large-sized transportation network. Section 6 contains our computational experience, based upon data in the Albany district of New York State. This experience illustrates the efficiency and effectiveness of our model and summarizes our computational results. Finally, Section 7 provides concluding remarks and suggestions for future research.

\section{Literature Review}

In this section, we briefly review the relevant literature in the area of hazmat routing, equity modeling and dissimilar path generation.

\section{1 hazmat Routing}

The research on hazardous material transportation problems focuses on two main issues: risk assessment and effective routing so as to mitigate risk. Much work has been done in risk modeling with a focus on probabilistic distribution of the risk over given areas: considering the risk related to the types of hazmat [2], using Geographic Information Systems [42], measuring the impact area of hazmat accidents [5], studying various transport modes [2], and assuming the accident probabilities known [33] or unknown [6].

Some prevailing studies emphasize the risk parameters to be measured during the transportation, including minimizing population exposure [35], expected risk [4], maximal risk [14], probability [36] or conditional probability [37], mean-variance of risk [14], and risk disutility [14]. Recently researchers have focussed on other considerations like transportation cost or risk equity dispersion [17]. Some bi-level hazmat transportation models have been developed to study the trade-off between the two conflicting objectives of minimizing transportation cost and risk of the hazmat transport 
$[23,30,40]$. Another recent direction of study is on building a model which has less reliance on the accuracy of historical accident data. An example of this is the VaR model developed in Kang et al. $[22]$.

The other main issue is route planning of hazmat trips, which involves a selection among the alternative paths between O-D pairs. Carriers usually focus on the routing problem of singlecommodity single O-D pair trips individually — to select a route between a given O-D pair for a given hazmat type - which is called local route planning [7]. However, the main concern of a government authority is to control the total risk over the population and the equity distribution of this risk over population zones, which can only achieved by simultaneously considering all of the local route planning problems, i.e. through global route planning. Examples of this approach for a single O-D pair and multiple trips of a single hazmat type are papers by Gopalan et al. [17] and Lindner-Dutton et al. [28].

While government control problems are usually modeled as bi-level optimization problems, we provide a single-level formulation in this paper. Our model can provide desirable paths for multiple hazmat and multiple O-D pairs. When a central authority can control all shipments via direct enforcements, carriers will follow the obtained paths. On the other hand, if carriers are autonomous decision making units over whom the central authority has no control, the obtained paths will provide guidelines for the central authority's action in the next step and our model does not provide how one can achieve the obtained paths. In such cases, one would need a bi-level optimization model, which may be formulated by extending the single-level formulation of this paper, to adequately model independent decision-making behavior of carriers. Heuristic solution approaches for such bi-level problems often requires a model and a solution method for the single-level problem. Therefore, this paper provides fundamental results that are useful to both cases directly and indirectly.

\subsection{Equity Modeling}

The concept of risk equity is well defined in Keeney [24], who defined equity as the largest difference in the risk level among a set of individuals. Several models have been proposed for addressing equity in the context of hazmat transport. Gopalan et al. [16] develop a model for a single hazmat trip in which the objective is to minimize risk subject to a set of constraints that ensure that the difference in risk borne by population zones is less than a set threshold (equity specification). This model was 
later generalized by Gopalan et al. [17] to the case of multiple hazmat trips of a single O-D pair. For this situation, Gopalan et al. [17] were able to show empirically that high level of equity can be achieved by modest increase in risk level.

Another method of enforcing equity is to limit the risk associated with a population zone or link. Current and Ratick [9] take such an approach by minimizing the maximum risk for a zone. Carotenuto et al. [8] focus on minimizing the total risk while constraining the risk on each traversed link. Both Current and Ratick [9] and Carotenuto et al. [8] consider a single hazmat trip. Our model is much more general than those proposed above. We allow multiple O-D pairs and multiple trips for each O-D pair. Our method of modeling equity is the same as that used in Gopalan et al. [16] and Gopalan et al. [17]. The other difference is that we use minimization of VaR as our objective. However, our approach can also be used with any other risk measures, including traditional risk as used in the majority of the literature.

\subsection{Dissimilar Path Generation}

The concept of dissimilar paths has been put forward in several contexts other than that of hazmat trips. Kuby et al. [25] apply dissimilar paths to reduce the search space for path-based models in a large, capacitated, multi-commodity network flow model. Lombard and Church [29] suggest generating a number of topologically dissimilar paths to avoid the repeated attempt of infeasible or undesirable paths, in the case of restricted layout problems like the corridor location application.

In the hazmat transport problem, the generation of spatially dissimilar paths is necessary to spread the risk equitably all over the network whenever multiple hazmat shipments are transported from an origin to a destination, and to provide more meaningful alternatives when the "best" path choice is not allowed in varied environments like bad weather conditions. There have been many methods to generate $k$ shortest paths, like Yen [41]. However, Yen's $k$-shortest path algorithm makes route choices based on transportation distance, which results in the spatial similarity among the generated paths. This situation is rather undesirable in hazmat route choices, where the objective is to reduce transportation risk more than transportation cost and highly overlapped road segments would severely increase the consequences of traffic accidents. To overcome this problem, a "p-dispersion" model is proposed by Kuby [26] to maximize the minimum dissimilarity of the paths on a general network. Erkut et al. [15] described and made an empirical comparison of ten 
heuristics for the discrete $p$-dispersion problem. Akgün et al. [3] present an improvement by selecting a subset from a large set of candidate paths and use a dispersion model to maximize the minimum dissimilarity among the paths in the subset, and compare their computational result with three other methods, the Iterative Penalty Method [21], the Gateway Shortest Path Method [29] and Minimax method [25]. Duarte and Marti [11] proposed and solved a similar maximum diversity problem with a constructive semi-greedy algorithm and a tabu search method.

Though the above methods have clearly defined the path dissimilarity, their work are restricted to single criterion, the edge length, and only consider the edges in the definition of dissimilarity. Dell'Olmo et al. [10] developed the dissimilarity path problem from a multi-objective perspective and proposed a multi-criteria shortest path algorithm (MSPA) to generate a set of non-dominated paths with the multiple objectives of length and risk. Thyagarajan et al. [38] consider both spatial and temporal information to determine dissimilar paths for military aircraft during mission ingress. Later on, Martí et al. [31] developed a modified Greedy Randomized Adaptive Search Procedure (GRASP) (See Resende and Ribeiro [34]) heuristic for a bi-objective path dissimilarity problem by making the trade-off between the two conflicting objectives: minimizing the average length of the paths while maximizing the dissimilarity among the paths.

In our model, we apply the dissimilar path concept to reduce the search space of the generalized hazmat VaR model. The generation of the $p$ disperse paths can be generated by any of the above methods. The main difference of our model lies in the generation of the candidate set - the $k$ minimal VaR paths, which cannot be converted into the $k$ shortest path problem.

\section{Formulation}

Consider a transport network $G=(\mathcal{N}, \mathcal{A})$, where $\mathcal{N}$ represents the node set, and $\mathcal{A}$ represents the link set. Define $\mathcal{I}$ as the O-D pair set within this network and $\mathcal{H}$ as the set of hazmat types. For each O-D pair $i \in \mathcal{I}$, there is a set of candidate paths $\mathcal{C}_{i}$. Suppose we have a set of trips $\mathcal{S}$ in the network $G$. Each trip $s \in \mathcal{S}$ is defined as a trip on an O-D pair $i \in \mathcal{I}$ with a type of hazmat $h \in \mathcal{H}$. The risk carried by the same route $j \in \mathcal{C}_{i}$ may be different with different kinds of hazmat. Given a risk confidence level $\alpha \in(0,1)$, the objective of our problem is to find a set of paths to minimize the total VaR over the set of trips $\mathcal{S}$ while incorporating VaR equity. To write the VaR equity 
constraint, we assume that the geographical region defined by the network $G$ can be divided into a set of mutually disjoint zones $\mathcal{Z}$. We define a zone pair ordering as an ordered pair $(a, b)$ of zones $a \in \mathcal{Z}$ and $b \in \mathcal{Z}$. The risk equity constraint is to maintain the difference in total VaR between each zone pair within a set threshold $\mu$. Table 1 presents the mathematical notation used in this paper.

In hazmat VaR models, the VaR of each path $j \in \mathcal{C}_{i}$ of O-D pair $i \in \mathcal{I}$ carrying a kind of hazmat $h \in \mathcal{H}$ is defined as

$$
\operatorname{VaR}_{\alpha i}^{j h}=\min \left\{\beta: \operatorname{Pr}\left\{R_{i}^{j h}>\beta\right\} \leq 1-\alpha\right\}
$$

Given a set of candidate paths $\mathcal{C}_{i}$, the corresponding hazmat shipment optimization problem is to solve:

$$
\operatorname{VaR}_{\alpha i}^{* h}=\min \left\{\mathrm{VaR}_{\alpha i}^{j h}: j \in \mathcal{C}_{i}, h \in \mathcal{H}\right\}
$$

Modeling the risk of traveling path $j$ by an accident probability $p_{u v}^{h}$ and an accident consequence $c_{u v}^{h}$ for each arc $(u, v) \in j$ and hazmat type $h$, Kang et al. [22] found that the optimal VaR value $\operatorname{VaR}_{\alpha i}^{* h}$ takes a value in the set $\{0\} \cup\left\{c_{u v}^{h}:(u, v) \in j, j \in \mathcal{C}_{i}\right\}$.

Hazmat VaR model is a new risk model different from any traditional hazmat risk models or finance VaR models. It is different from other hazmat risk model in that it brings the factor of confidence levels into the risk model and provides a set of varying route solutions according to decision makers' risk preferences. Though VaR is a well-known concept in finance field, the definition of $\mathrm{VaR}$ in hazmat transportation is different than VaR in finance. First, in hazmat VaR model, the measurement units of investment (a route) and measurement object (risk) are inconsistent. Besides, different from finance VaR model, the component of investment, each road segment along the route of a shipment is dependent on their order and their risks are non-additive. Finally, evaluating the VaR value of each path and solving the VaR minimization model for hazmat transportation have unique characteristics and require more complicated methods than those for financial VaR problems.

Let $x_{\alpha i}^{j h}$ be an integer decision variable, representing the volume of hazmat type $h \in \mathcal{H}$ on path $j \in \mathcal{C}_{i}$ for O-D pair $i \in \mathcal{I}$ given confidence level $\alpha$, where $\mathcal{C}_{i}$ is the set of all paths between the O-D pair $i$. A formulation for the generalized hazmat VaR routing problem is as follows:

$$
\text { (P) } \quad \min _{x} \sum_{h \in \mathcal{H}} \sum_{i \in \mathcal{I}} \sum_{j \in \mathcal{C}_{i}} \beta_{\alpha i}^{j h} x_{\alpha i}^{j h}
$$




\section{Table 1: Mathematical Notation Table}

\begin{tabular}{|c|c|}
\hline Notation & Definition \\
\hline \multicolumn{2}{|l|}{ Sets } \\
\hline$G(\mathcal{N}, \mathcal{A})$ & a graph of road network \\
\hline $\mathcal{N}$ & set of nodes, $|\mathcal{N}|=n$ \\
\hline $\mathcal{A}$ & set of links, $|\mathcal{A}|=m$ \\
\hline $\mathcal{I}$ & Set of O-D (Origin-Destination) pairs \\
\hline $\mathcal{S}$ & Set of trips \\
\hline $\mathcal{H}$ & Set of hazmat types \\
\hline $\mathcal{Z}$ & Set of geographical zones in $G$ \\
\hline $\mathcal{C}_{i}$ & Set of available paths for O-D pair $i \in \mathcal{I}$ \\
\hline $\mathcal{M}_{\alpha i}^{h k}$ & $\begin{array}{l}\text { Set of } k \text {-minimal VaR paths for O-D pair } i \in \mathcal{I} \text { and hazmat type } h \in \mathcal{H} \text { under confidence level } \alpha \text {, } \\
\mathcal{M}_{\alpha i}^{h k} \subseteq \mathcal{C}_{i}\end{array}$ \\
\hline $\mathcal{P}_{\alpha i}^{h}$ & Set of dissimilar paths for O-D pair $i \in \mathcal{I}, \mathcal{P}_{\alpha i}^{h} \subseteq \mathcal{M}_{\alpha i}^{h k}$ \\
\hline \multicolumn{2}{|c|}{ Parameters } \\
\hline$\alpha$ & confidence level to control the worst risk brought by hazmat transportation \\
\hline$\delta_{u v}$ & length of link $(u, v)$ \\
\hline$p_{u v}^{h}$ & accident probability in link $(u, v)$ for hazmat type $h$ \\
\hline$c_{u v}^{h}$ & accident consequence from an accident in link $(u, v)$ for hazmat type $h$ \\
\hline $\mathrm{VaR}_{\alpha i}^{j h}$ & cutoff risk for a path $j$ given confidence level $\alpha$ \\
\hline$\beta_{\alpha i}^{j h}$ & $\begin{array}{l}\text { VaR value brought by a trip with hazmat type } h \in \mathcal{H} \text { on the path } j \in \mathcal{C}_{i} \text { of O-D pair } i \in \mathcal{I} \text { given } \\
\text { confidence level } \alpha\end{array}$ \\
\hline$\hat{\beta}_{\alpha i}^{j h}(z)$ & $\begin{array}{l}\text { VaR value brought by a trip with hazmat type } h \in \mathcal{H} \text { on the path } j \in \mathcal{C}_{i} \text { of O-D pair } i \in \mathcal{I} \text { to a } \\
\text { geographical zone } z \in \mathcal{Z} \text { given confidence level } \alpha\end{array}$ \\
\hline & number of trips assigned to O-D pair $i \in \mathcal{I}$ with hazmat type $h \in \mathcal{H}$ \\
\hline$D_{j_{1} j_{2}}$ & dissimilarity index between two candidate paths $j_{1}$ and $j_{2}$ \\
\hline$\mu$ & threshold for risk equity constraint \\
\hline$\eta$ & Lagrangian multipliers \\
\hline \multicolumn{2}{|l|}{ Variables } \\
\hline$R_{i}^{j h}$ & risk brought by path $j \in \mathcal{C}_{i}$ with O-D pair $i \in \mathcal{I}$ carrying hazmat $h \in \mathcal{H}$ \\
\hline$x_{\alpha i}^{j h}$ & $\begin{array}{l}\text { number of trips assigned to path } j \in \mathcal{C}_{i} \text { selected for trip with hazmat type } h \in \mathcal{H} \text { on O-D pair } i \in \mathcal{I} \\
\text { or not given confidence level } \alpha\end{array}$ \\
\hline$y_{\alpha i}^{j h}$ & binary variable indicating whether path $\mathrm{j}$ is selected into the dissimilar path subset $\mathcal{P}_{\alpha i}^{h}$ \\
\hline
\end{tabular}

subject to

$$
\begin{aligned}
\sum_{j \in \mathcal{C}_{i}} x_{\alpha i}^{j h}=n_{h i}, \quad \forall i \in \mathcal{I}, \quad \forall \quad h \in \mathcal{H} \\
\sum_{h \in \mathcal{H}} \sum_{i \in \mathcal{I}} \sum_{j \in \mathcal{C}_{i}}\left(\hat{\beta}_{\alpha i}^{j h}(a)-\hat{\beta}_{\alpha i}^{j h}(b)\right) x_{\alpha i}^{j h} \leq \mu, \quad \forall \quad a, b \in \mathcal{Z} \\
x_{\alpha i}^{j h} \text { integer, } \quad \forall \quad j \in \mathcal{C}_{i} \quad \forall \quad i \in \mathcal{I} \quad \forall h \in \mathcal{H}
\end{aligned}
$$

where $\beta_{\alpha i}^{j h}$ denotes the maximum cutoff risk brought by hazmat $h \in \mathcal{H}$ along path $j \in \mathcal{C}_{i}$ of the O-D pair $i \in \mathcal{I}$ under confidence level $\alpha$, and $\hat{\beta}_{\alpha i}^{j h}(z)$ represents the maximum cutoff risk to zone $z$ brought by $h \in \mathcal{H}$ along $j \in \mathcal{C}_{i}$ given $\alpha$ for all zones $z \in \mathcal{Z}$. 
The objective of the generalized hazmat VaR routing problem is to minimize the cumulative VaR values of all hazmat trips in the road network $G$ given $\alpha$. The constraint set (4) constrains the number of trips on O-D pair $i \in \mathcal{I}$ carrying hazmat type $h \in \mathcal{H}$. The constraint set (5) represents the risk equity constraints. Here $\hat{\beta}_{\alpha i}^{j h}(z)$ represents the cumulative VaR value to a zone $z$ given $\alpha$. We note that ordered zone pair $(a, b)$ is different from $(b, a)$, and we have no a priori knowledge about which zone pair sustains the maximum risk difference for traveling on an arbitrary path. Therefore, it is necessary to compare the VaR dissimilarity for each zone pair ordering $(a, b)$ rather than a random selection of a pair of zones. Finally constraint set (6) restricts $x_{\alpha i}^{j h}$, the number of trips with hazmat type $h \in \mathcal{H}$ on each path $j \in \mathcal{C}_{i}$ of an O-D pair $i \in \mathcal{I}$, as integer.

The generalized hazmat VaR routing model is difficult to solve due to the risk equity constraint (5). If this constraint is absent, then the problem can be decomposed into separate routing problems for each hazmat and origin-destination combination, each of which can be solved using the algorithm in Kang et al. [22]. There are many different ways to obtain an equitable set of routes. For example, Gopalan et al. [17] bound the maximum risk sustained by any zone within a set threshold. This constraint offers the advantage of being able to enforce equity between those zones that provide reasonable transport path alternatives. We adopt this risk equity formulation in our model to spread the risk into different zones, but with a different definition of the risk equity parameters $\hat{\beta}_{\alpha i}^{j h}(z)$. In our case, the risk of a trip to a zone is the maximal cutoff risk that its path will experience within that zone under the given confidence level $\alpha$. After segmenting the road segments of the path in each zone, we can calculate the zone $\operatorname{VaR}$ value $\hat{\beta}_{\alpha i}^{j h}(z)$ with the same method for path VaR value $\beta_{\alpha i}^{j h}$. The detailed calculation method has been described in Kang et al. [22].

\section{Determination of Candidate Paths}

For the problem $(P)$ to deliver an optimal solution to the hazmat routing problem, every possible path between every O-D pair must be included. This leads to an unmanageable formulation that requires path enumeration. To circumvent this difficulty we study in this section the problem of finding a reasonable set of paths for each trip for inclusion in the optimization problem $(P)$. To restate, two objectives are considered in this model. One is still minimizing the total VaR value, and the other is the risk equity, i.e., dispersing traffic flows to reduce the accumulative consequences 
brought by busy road segments. In other words, we wish to select routes with as small VaR value as possible but also as geographically dissimilar as possible. With this in mind, we provide a two-stage candidate paths preprocessing procedure. First we reduce the candidate paths for the trip to the first $k$ minimal VaR paths. Second, from the $k$ minimal VaR paths, we further reduce the candidates to a reasonable number of dispersed paths.

\subsection{Single Trip Case}

We start by assuming that there are a number of feasible routes available for a given O-D pair $i \in \mathcal{I}$, say, set $\mathcal{C}_{i}$. Each path in the set $\mathcal{C}_{i}$ represents an option that is acceptable to the carrier for routing a certain hazmat between the O-D pair. Suppose that for each trip $s \in \mathcal{S}$ with the O-D pair $i \in \mathcal{I}$ carrying a hazmat type $h \in \mathcal{H}$, we are able to select a candidate set $\mathcal{M}_{\alpha i}^{h k} \subseteq \mathcal{C}_{i}$ containing $k$-minimal VaR paths given $\alpha$. That is, the set $\mathcal{M}_{\alpha i}^{h k}$ contains the path with the smallest VaR to the path with $k$-th smallest VaR for the trip $s \in \mathcal{S}$ under the confidence level $\alpha$.

From the candidate set $\mathcal{M}_{\alpha i}^{h k}$, we will determine a subset $\mathcal{P}_{\alpha i}^{h} \subseteq \mathcal{M}_{\alpha i}^{h k}$ for each O-D pair such that every pair of paths in the subset $\mathcal{P}_{\alpha i}^{h}$ are as dissimilar from each other as possible. These candidate routes represent routes (set of links that connect a particular O-D pair) that are spatially dissimilar to one another (dissimilar with respect to a distance metric). By selecting a subset of dissimilar paths from the $\mathrm{k}$ shortest path set $\mathcal{M}_{\alpha i}^{h k}$, we limit the usage of similar paths from consideration and mitigate the consequence caused by trip route centralization.

$k$-Minimal VaR Paths Determination The $k$-minimal VaR path set $\mathcal{M}_{\alpha i}^{h k}$ is a variant of the $k$-shortest path, which aims to sort and select from the available path set for a trip from origin (O) to destination (D) with defined criteria, shortest distance or minimal risk. In the $k$-minimal hazmat VaR transportation problem, we intend to find the first $k$ minimal VaR paths. $\mathcal{M}_{\alpha i}^{h k}$ can be constructed in many different ways. But generally speaking, no matter how those algorithms vary, they can be divided into two categories: Removing Path algorithm and Deviation Path algorithm. The Removing Path algorithm is proposed by Martins [32]. In this algorithm, in each iteration $k$, the graph is reconstructed by removing the first $1, \cdots, k-1$ paths while keeping the new graph equivalent to the original graph. That means, any path between two nodes in the original graph can be found in the new graph too. Then search the $k$-th shortest path in the original graph equals 
to the shortest path problem and can be solved by classic shortest path algorithms like Dijkstra's. The Deviation Path algorithm is proposed by Eppstein [12], whose main idea of this algorithm is to obtain the $k$-shortest path $j^{k}$ from a set of candidate paths composed by the deviation paths of $j^{1}, j^{2}, \cdots, j^{k-1}$.

The Deviation Path algorithm Yen [41] has proved to be an effective method to solve the $k$-shortest path problem, as it does not need to reconstruct graphs. However, the efficiency of the Deviation Path algorithm is built based on the construction of a partial search space for the $(k+1)$-st path - the set of deviation paths from the neighborhood of the $k$-th best path. Thus, it is not necessarily effective in solving problems with objectives other than transportation cost. In particular, for the $k$-minimal VaR path problem, the 2nd minimal VaR path is not necessarily contained in the neighborhood of the 1st minimal VaR path. By contrast, the Removing Path algorithm is much more effective in such a situation. Given a certain confidence level $\alpha$, by removing the 1st minimal VaR path from the network $G^{1}$, we can always find the 2 nd minimal VaR path from the resulting graph $G^{2}$. Similarly, by removing the 2 nd, 3rd, $\cdots,(k-1)$-st paths, the $k$-th minimal VaR path can be generated from the newly constructed network $G^{k}$. The $k$ minimal VaR path set $\mathcal{M}_{\alpha i}^{h k}$ can be defined as the set of minimal VaR paths in each newly constructed graph $G^{k}$ for O-D pair $i \in \mathcal{I}$,

$$
\mathcal{M}_{\alpha i}^{h k}=\left\{j^{k}: \operatorname{VaR}_{\alpha i}^{h k}=\min _{j \in Q} \operatorname{VaR}_{\alpha i}^{j h}, \quad \text { where } \quad Q \in \mathcal{C}_{i} \backslash\left\{j^{1}, \cdots, j^{k-1}\right\}\right\}
$$

Dis/similarity Index Definition and Spatially Dissimilar Paths Selection With the $k$ minimal VaR path set $\mathcal{M}_{\alpha i}^{h k}$ determined for a trip carrying hazmat type $h$ with O-D pair $i$, we can select the dissimilar VaR path subset $\mathcal{P}_{\alpha i}^{h}$ with any method in the literature review. We have discussed many methods in Section 2.3 to solve the dissimilar path problem in different ways, with each of them having a similarity or dissimilarity function. Our model utilizes the $p$-dispersion method proposed by Akgün et al. [3] to generate the $\left|\mathcal{P}_{\alpha i}^{h}\right|$ spatially dissimilar paths.

Define $D_{j_{1} j_{2}}$ to be the distance between candidate paths $j_{1}$ and $j_{2}$. The $\left|\mathcal{P}_{\alpha i}^{h}\right|$-dispersion problem can be expressed as follows:

$$
\max _{\mathcal{P}_{\alpha i}^{h} \subseteq \mathcal{M}_{\alpha i}^{h k}} \min _{\substack{j_{1} \neq j_{2} \\ j_{1}, j_{2} \in \mathcal{P}_{\alpha i}^{h}}} D_{j_{1} j_{2}}
$$


In our problem, $D_{j_{1} j_{2}}$ represents the dissimilarity index between any pair of paths.

$$
D_{j_{1} j_{2}}=1-\frac{\left[d\left(j_{1}, j_{2}\right) / l_{j_{1}}+d\left(j_{1}, j_{2}\right) / l_{j_{2}}\right]}{2}
$$

where $l_{j}$ denotes the length of path $j$ and $d(\cdot, \cdot)$ denotes the length of the shared portion of two paths. The problem (8) can be converted into the following formulation:

$$
\max _{\mathcal{P}_{\alpha i}^{h} \subseteq \mathcal{M}_{\alpha i}^{h k}} D_{\mathcal{P}_{\alpha i}^{h}}
$$

subject to

$$
\begin{aligned}
& \sum_{j \in \mathcal{P}_{\alpha i}^{h}} y_{\alpha i}^{j h}=\left|\mathcal{P}_{\alpha i}^{h}\right| \quad \forall \mathcal{P}_{\alpha i}^{h} \subseteq \mathcal{M}_{\alpha i}^{h k} \\
& D_{\mathcal{P}_{\alpha i}^{h}} \leq D_{j_{1} j_{2}} y_{\alpha i}^{j_{1} h} y_{\alpha i}^{j_{2} h} \quad \forall j_{1}, \quad j_{2}>j_{1}, \quad j_{1}, j_{2} \in \mathcal{P}_{\alpha i}^{h}, \quad \forall \mathcal{P}_{\alpha i}^{h} \subseteq \mathcal{M}_{\alpha i}^{h k} \\
& y_{\alpha i}^{j h} \in\{0,1\} \quad \forall j \in \mathcal{P}_{\alpha i}^{h}
\end{aligned}
$$

The objective of the $p$-dispersion method is to maximize the minimum distance between any pair of the selected paths. Erkut [13] solved this problem in a two-phase heuristic, by constructing an initial solution as in a greedy-algorithm, and then searching locally to improve the initial solution. Akgün et al. [3] provided a detailed comparison with three methods that are commonly used: the Iterative Penalty Method, the Gateway Shortest Path Method and Minimax. They empirically demonstrate that the $p$-dispersion method is more effective in generating a set of dissimilar paths. However, a point worth noting is that, unlike the other three methods, the solution generated by the $p$-dispersion method does not necessarily include the least risk path. It may be inappropriate for any decision maker to accept a set of routes not including the best path. This drawback can be overcome by placing restrictions on the size of $\left|\mathcal{M}_{\alpha i}^{h k}\right|$ and $\left|\mathcal{P}_{\alpha i}^{h}\right|$, or by enforcing constraints on the risk threshold.

\subsection{Multiple Trip Case}

Consider a set of trips $\mathcal{S}$ to be shipped among O-D pairs $\mathcal{I}$, carrying different hazmat from materials set $\mathcal{H}$. There are $|\mathcal{S}|$ candidate sets of minimal VaR routes and $|\mathcal{S}|$ subsets of dissimilar 
VaR routes selected from them. Let these be labeled as $\mathcal{P}_{\alpha i(1)}^{h(1)}, \mathcal{P}_{\alpha i(2)}^{h(2)}, \cdots, \mathcal{P}_{\alpha i(|\mathcal{S}|)}^{h(|\mathcal{S}|)}$, where $\mathcal{P}_{\alpha i(1)}^{h(1)} \subseteq$ $\mathcal{M}_{\alpha i(1)}^{h(1) k}, \mathcal{P}_{\alpha i(2)}^{h(2)} \subseteq \mathcal{M}_{\alpha i(2)}^{h(2) k}, \cdots, \mathcal{P}_{\alpha i(|\mathcal{S}|)}^{h(|\mathcal{S}|)} \subseteq \mathcal{M}_{\alpha i(|\mathcal{S}|)}^{h(|\mathcal{S}|) k}$. The key problem is how to generate the $k-$ minimal VaR path subsets $\mathcal{M}_{\alpha i(|\mathcal{S}|)}^{h(|\mathcal{S}|) k}, \mathcal{M}_{\alpha i(|\mathcal{S}|)}^{h(|\mathcal{S}|) k}, \cdots, \mathcal{M}_{\alpha i(|\mathcal{S}|)}^{h(|\mathcal{S}|)}$ for each trip $s \in \mathcal{S}$. Once the first $k$ VaR candidate path sets $\mathcal{M}_{\alpha i(s)}^{h(s) k}$ s are generated, the dissimilar path subset $\mathcal{P}_{\alpha i(1)}^{h(1)}, \mathcal{P}_{\alpha i(2)}^{h(2)}, \cdots, \mathcal{P}_{\alpha i(|\mathcal{S}|)}^{h(|\mathcal{S}|)}$ can be selected from candidate set $\mathcal{M}_{\alpha i(1)}^{h(1) k}, \mathcal{M}_{\alpha i(2)}^{h(2) k}, \cdots, \mathcal{M}_{\alpha i(|\mathcal{S}|)}^{h(|\mathcal{S}|) k}$.

General Procedure to Determine Geographically Dissimilar VaR Paths Our solution to find dissimilar VaR paths for a hazmat trip can be described as a two-step solution:

1. Constructing the Candidate Set of Minimal VaR Paths Using Removing Paths Procedure

(a) Remove Paths Procedure

Step 0: Assuming the shortest path $l=\left\{s_{1},\left(s_{1}, s_{2}\right), \cdots, s_{m-1},\left(s_{m-1}, s_{m}\right), s_{m}=s_{n}\right\}$, set $f l a g=d\left(s_{1}, s_{2}\right)$;

Step 1: Delete $\left(s_{1}, s_{2}\right)$ from $G(N, A)$;

- $\quad$ If $s_{2}=s_{n}$, stop;

- $\quad$ otherwise, go to Step 2;

Step 2: $\forall j=2, \cdots, m-1$, adjoin to $N$ a node $s_{j}^{\prime}$;

Step 3: $\forall j=2, \cdots, m-1$, and $\forall\left(s_{j}, x\right) \in A$ such that $x \neq s_{j+1}$, adjoin $\left(s_{j}^{\prime}, x\right)$ to $A$ and set $d\left(s_{j}^{\prime}, x\right)=d\left(s_{j}, x\right)$;

Step 4: $\forall j=2, \cdots, m-2$, adjoin $\left(s_{j}^{\prime}, s_{j+1}^{\prime}\right)$ to $A$ and set $d_{i+1}\left(s_{j}^{\prime}, s_{j+1}^{\prime}\right)=d\left(s_{j}, s_{j+1}\right)$;

Step 5: Adjoin $\left(s_{1}, s_{2}^{\prime}\right)$ to $A$ and set $d\left(s_{1}, s_{2}^{\prime}\right)=$ flag.

(b) Minimal VaR Path Algorithm

Step 0: Set $k=1$;

Step 1: Determine the shortest path $l_{k}^{\prime}$ in $G_{k}\left(N_{k}, A_{k}\right)$;

- $\quad$ If $l_{k}^{\prime}$ is not found in $G_{k}\left(N_{k}, A_{k}\right)$, stop, all paths have been determined;

- $\quad$ Otherwise, identify $l_{k}^{\prime}$ with its corresponding path $l_{k}$ in $G_{1}\left(N_{1}, A_{1}\right)-l_{k}$, as the $k$ th shortest path in $G_{1}\left(N_{1}, A_{1}\right)$;

Step 2: If $k=\left|\mathcal{M}_{\alpha}\right|$, finish the algorithm; Otherwise go to step 3; 
Step 3: Use one of the stated procedures to delete $l_{k}^{\prime}$ from $G_{k}\left(N_{k}, A_{k}\right)$, to obtain $G_{k+1}\left(N_{k+1}, A_{k+1}\right)$

Step 4: Set $k=k+1$, return to Step 1 .

2. Selecting Spatially Dissimilar VaR Paths

(a) Define a dissimilarity index between any pair of paths.

$$
D_{j_{1} j_{2}}=1-\frac{\left[d\left(j_{1}, j_{2}\right) / l_{j_{1}}+d\left(j_{1}, j_{2}\right) / l_{j_{2}}\right]}{2}
$$

where $l_{j}$ denotes the length of path $j$ and $d(\cdot, \cdot)$ denotes the length of the shared portion of two paths.

(b) $p$-Disperse Algorithm

Step 0: Initialization. $k=0, p$-disperse path queue $T_{0}=\left\{j_{0}\right\}$ with the ascending VaR order, $j_{0}$ is the minimal VaR path, left path queue $\bar{T}_{0}=\mathcal{M}-T_{0}$, set $\max \min D=0$;

Step 1: Loop. In iteration $k$, extract path $j_{k}$ from $\bar{T}_{k}$, calculate the dissimilarity index between $j_{k}$ and all paths in $T_{k}$, get minimal distance $\min D_{k}$.

Step 2: Storing the incumbent. If $\max \min D<\min D_{k}, \max \min D \leftarrow \min D_{k}, T \leftarrow$ $T \bigcup\left\{j_{k}\right\}$

Step 3: Stopping rule. Stop if $\bar{T}=\emptyset$ or $k=|\mathcal{P}|$. Else $k \leftarrow k+1$, go to step1.

\section{$5 \quad$ Lagrangian Relaxation Solution Method}

With the VaR value of each path and risk equity parameters of each path in each zone determined, the problem can be regarded as an integer programming problem. It can be easily solved with software like CPLEX12.1 for small- or medium-sized road network. Table 2 displays the results of our computational experiments with Java1.6+CPLEX12.1 on a $3.40 \mathrm{GHz}$ CPU, 2.00GB RAM computer system:

In order to solve the hazmat VaR problem on a large-sized road network, we apply a branch and bound procedure, converting this problem into a simple traffic flow assignment problem by relaxing 
Table 2: Computational Results From Data Sets By CPLEX

\begin{tabular}{llllll}
\hline $\begin{array}{l}\text { O-D } \\
\text { Pairs }\end{array}$ & $\begin{array}{l}\text { Paths for } \\
\text { Each O-D Pair }\end{array}$ & $\begin{array}{l}\text { Hazmat } \\
\text { Types }\end{array}$ & $\begin{array}{l}\text { Geographical } \\
\text { Zone }\end{array}$ & $\begin{array}{l}\text { Presolve Time } \\
\text { (seconds) }\end{array}$ & $\begin{array}{l}\text { Total } \\
\text { Time }\end{array}$ \\
\hline 1 & 1 & 1 & 2 & 0.00 & 0.01 seconds \\
2 & 2 & 2 & 2 & 0.00 & 0.01 seconds \\
10 & 10 & 10 & 10 & 0.01 & 2 seconds \\
20 & 20 & 20 & 20 & 0.02 & 4 minutes 24 seconds \\
30 & 30 & 30 & 30 & 0.03 & 102 minutes 2 seconds \\
\hline
\end{tabular}

the risk equity constraint in a Lagrangian manner, and appending them to the objective function with Lagrangian multipliers (penalties) $\eta_{a b}$. We obtain the following Lagrangian relaxation problem:

$$
g(\eta)=\min _{x} \sum_{h \in \mathcal{H}} \sum_{i \in S} \sum_{j \in \mathcal{P}_{\alpha i}^{h}} \beta_{\alpha i}^{\prime j h} x_{\alpha i}^{j h}-\sum_{a \in \mathcal{Z}} \sum_{b \in \mathcal{Z}} \mu \eta_{a b}
$$

subject to

$$
\begin{aligned}
& \sum_{j \in \mathcal{P}_{\alpha i}^{h}} x_{\alpha i}^{j h}=n_{h i}, \quad \forall i \in \mathcal{I}, \quad \forall \quad h \in \mathcal{H} \\
& x_{\alpha i}^{j h} \text { integer, } \quad \forall \quad j \in \mathcal{P}_{\alpha i}^{h} \quad \forall \quad i \in \mathcal{I} \quad \forall h \in \mathcal{H}
\end{aligned}
$$

where $\beta_{\alpha i}^{\prime j h}=\beta_{\alpha i}^{j h}+\sum_{a \in \mathcal{Z}} \sum_{b \in \mathcal{Z}} \eta_{a b}\left\{\hat{\beta}_{\alpha i}^{j h}(a)-\hat{\beta}_{\alpha i}^{j h}(b)\right\}$.

With given Lagrangian multipliers, the relaxed problem reduces to a simple assignment problem and can be easily solved by picking $j$ with the minimum $\beta_{\alpha i}^{\prime j h}$ value, setting $x_{\alpha i}^{j h}$ to $n_{h i}$, and all other variables $x_{\alpha i}^{l h}$ such that $l \neq j, l \in \mathcal{P}_{\alpha i}^{h}$ to 0 . Usually Lagrangian relaxation can be used to generate a lower bound for the integer programming problem. However, the Lagrangian relaxation of our hazmat trip problem has the integrality property, that means, a solution to the Lagrangian relaxation is naturally integral. Therefore, the Lagrangian formulation can be replaced by the linear programming relaxation. In our solution procedure, we utilize the linear programming relaxation of the original problem (3) as a lower bound, while applying Subgradient Search Algorithm [19] to obtain an upper bound for our problem. Whenever the solution obtained in the Lagrangian relaxation scheme is feasible to the original problem, we compute its objective function value with the original objective coefficients and see if it provides an improved upper bound, i.e. it is better than the best solution found so far. The most widely used and successful methods for finding 
improved multipliers use the concepts of gradient and subgradient search. At iteration 0, we start with all multipliers $\eta_{a b}^{0}$ being zero for all $a, b \in \mathcal{Z}$, and at each iteration $r>1$, update them as

$$
\eta_{a b}^{r+1}=\eta_{a b}^{r}+\lambda_{r} \times \frac{\sum_{a \in \mathcal{Z}} \sum_{b \in \mathcal{Z}} \eta_{a b}^{r}\left\{\pi_{a}^{h}(i, j)-\pi_{b}^{h}(i, j)\right\}-\mu}{\left\|\sum_{a \in \mathcal{Z}} \sum_{b \in \mathcal{Z}} \eta_{a b}^{r}\left\{\pi_{a}^{h}(i, j)-\pi_{b}^{h}(i, j)\right\}-\mu\right\|}
$$

where $\left\{\lambda_{r}\right\}$ is a sequence such that

$$
\begin{aligned}
\lambda_{r} & \geq 0 \quad \forall r \\
\lim _{r \rightarrow \infty} \lambda_{r} & =0 \\
\sum_{r=1}^{\infty} \lambda_{r} & =\infty
\end{aligned}
$$

Our computational analysis in Section 6.2 demonstrates that for large-sized network as $1000 \times$ $10 \times 10 \times 10$ O-D pair $\times$ candidate paths $\times$ hazmat types $\times$ zones, the Lagrangian relaxation solution can solve the problem within 5 minutes, with a gap within $0.5 \%$ from the LP bound.

In summary, the solution to the generalized Hazmat VaR problem can be summarized in the following flowchart Figure 1:

\section{Case Study and Experimental Analysis}

We provide results from our computational experience based on the data set obtained from the transportation network surrounding the county of Albany, New York. We chose this region because it is a key junction of major interstates and is a hub of hazmat transportation activity. The understudy area includes the Albany area and its neighborhoods of Rensselaer, Saratoga, and Montgomery, and involves seven interstate and US highways: I-90, I-890, I-87, I-787, US-20, US-9 and US-9W.

We applied a two segmentation strategy in order to test the affect of geographical zone segmentation to risk mitigation. We separately divide this network into 28 and 7 geographical zones according to the nearby townships in Albany County (See Figure A.1). The transportation network in Albany County was pruned to 90 nodes and 108 arcs. See Figure 2 for a map of the pruned network. Two types of hazmats were chosen for the purpose of risk estimation, Benzyl Chloride and Toluene. Different sets of data were developed, representing various combinations of O-D pairs, 


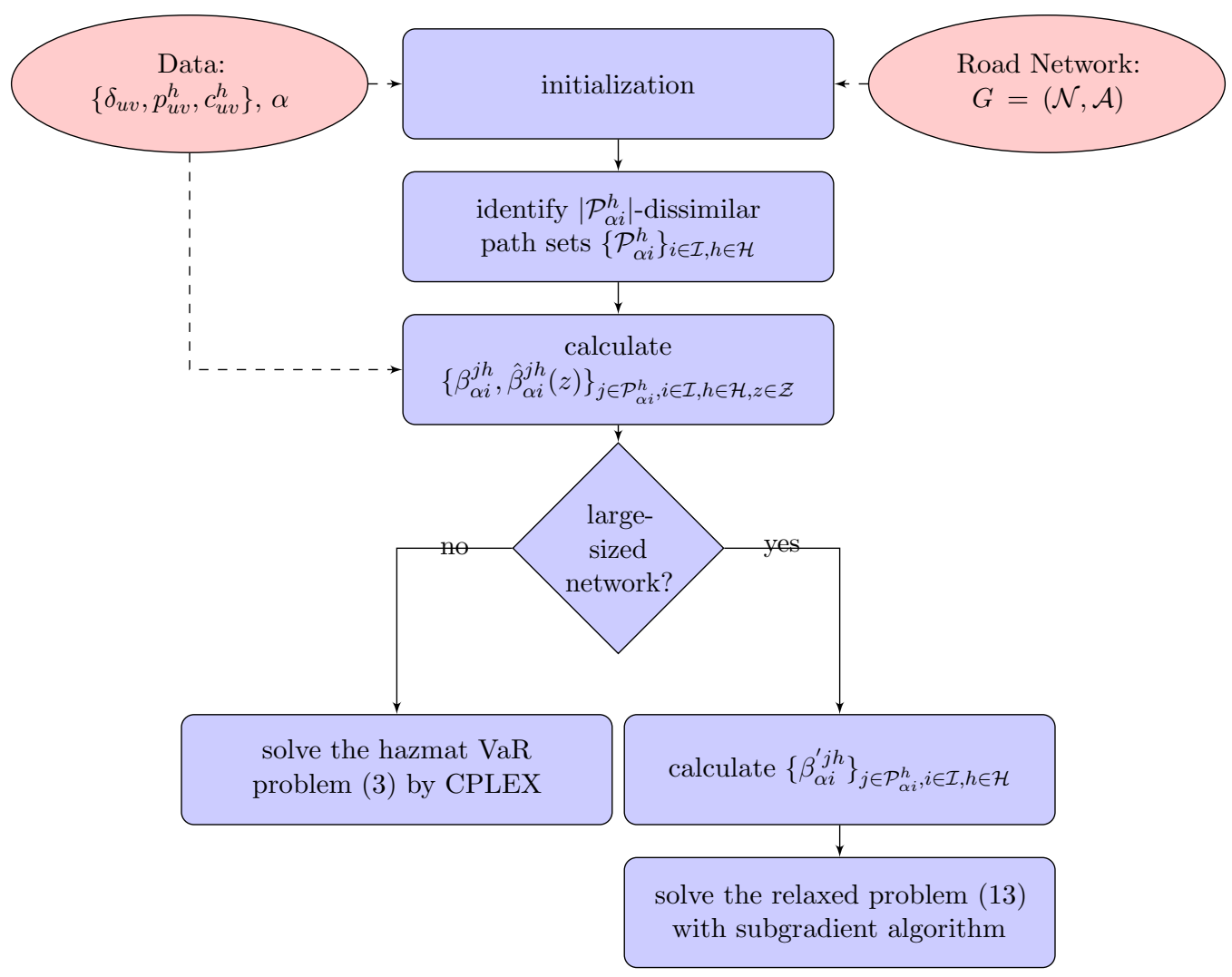

Figure 1: Generalized Hazmat VaR Problem Solution Flowchart

geographical zones, different types of hazardous materials, and assumed radius of spread considered.

The link accident probabilities are calculated according to road segment lengths as stated in Abkowitz and Cheng [1]:

$$
p=10^{-6} \times \delta
$$

where the road segment length $\delta$ is in miles.

The link consequences are calculated according to population density within the neighborhood around the links. With different radii of spread $\lambda$ from different hazmats, the endangered area can be described with a whole $\lambda$-neighborhood which is a concept developed by Batta and Chiu [5]. Here we simply compute the link consequence as the function:

$$
c=\left(\pi \lambda^{2}+2 \times \lambda \times \delta\right) \times \rho,
$$

where $\rho$ is the population density in the neighborhood along the road segment, measured in persons per mile-sq. We collected the road lengths and the population statistics from the websites of the 


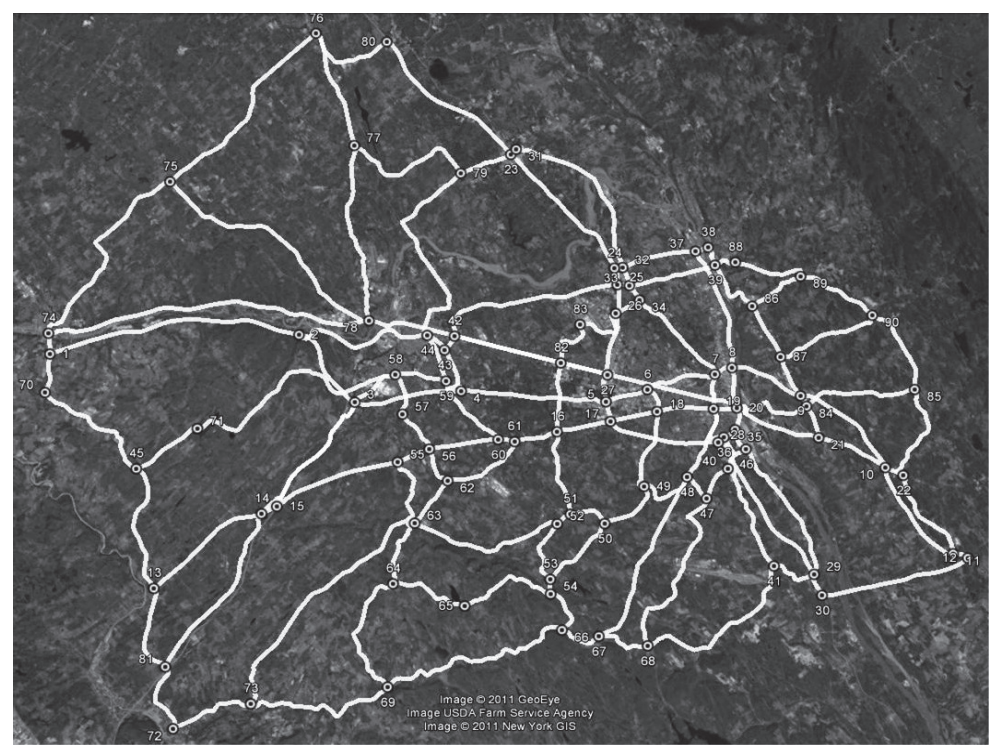

Figure 2: Albany Area Highway Roadmap

U.S. Department of Transportation and the U.S. Department of Commerce.

\subsection{Candidate Path Generation}

Generation of dissimilar candidate set $\mathcal{P}_{\alpha i}^{h}$ affects the computation quality and search speed of the route choices. We apply the modified $\left|\mathcal{P}_{\alpha i}^{h}\right|$-dispersion method [3] to generate $\left|\mathcal{P}_{\alpha i}^{h}\right|$ dissimilar paths out of the $\mathcal{M}_{\alpha i}^{h k}$ minimal VaR path set for each trip with O-D pair $i$ carrying hazmat $h$. We compare the effect of the size of the number of dissimilar paths selected, $\left|\mathcal{P}_{\alpha i}^{h}\right|$, and the size of the number of candidate dissimilar paths, $\left|\mathcal{M}_{\alpha i}^{h k}\right|$. To do this comparison, we use four indicators: Average VaR value (AvVaR), Average Length (AvLen), Average Dissimilarity (AvDi) and Minimal Dissimilarity (MiDi).

\subsubsection{Varying $\mathcal{P}_{\alpha i}^{h}$ for Fixed $\mathcal{M}_{\alpha i}^{h k}$}

We study the case of O-D pair (1,22). Table 3 displays the computational results for $\left|\mathcal{P}_{0.999995,(1,22)}^{\text {Benzyl Chloride }}\right|=$ $2,5,10,20$, from the same set of candidate path set $\mathcal{M}_{0.999995,(1,22)}^{\text {Benzl Chloride } k}$. The values of $\left|\mathcal{M}_{0.999995,(1,22)}^{\text {Benzyl Chloride } k}\right|$ considered are $k=10,20$ and 30 . For each candidate set $\mathcal{M}_{0.999995,(1,22)}^{\text {Benzyl Chloride, } k}$, we have the same basic result: We obtain better solutions when $\mathcal{P}_{0.999995,(1,22)}^{\text {Benzyl Chloride }}$ is smaller. 
Table 3: Computational Results for Dissimilar Hazmat Paths - VaR Model Under $\alpha=0.999995$ vs. TR Model, O-D Pair $(1,22)$

\begin{tabular}{|c|c|c|c|c|c|c|c|c|c|}
\hline$|\mathcal{M}|$ & $|\mathcal{P}|$ & MiVaR & $\begin{array}{l}\text { Rank } \\
\text { in } \mathcal{M}\end{array}$ & $\begin{array}{c}\text { Gap } \\
\text { (from Opt) }\end{array}$ & AvVaR & AvLen & AvDi & MiDi & $\begin{array}{l}\text { Time } \\
\text { (sec) }\end{array}$ \\
\hline \multicolumn{10}{|c|}{$\mathrm{VaR}_{0.999995}$ Model } \\
\hline \multirow{2}{*}{10} & 2 & 5571.92 & 1 & $0 \%$ & 6439.50 & 51.25 & 58.37 & 58.37 & 3 \\
\hline & 5 & 5571.92 & 1 & $0 \%$ & 6130.28 & 50.74 & 27.93 & 15.11 & 3 \\
\hline \multirow{2}{*}{20} & 2 & 5571.92 & 1 & $0 \%$ & 8420.46 & 48.6 & 78.19 & 78.19 & 10 \\
\hline & 10 & 5571.92 & 1 & $0 \%$ & 8303.45 & 51.35 & 32.46 & 15.16 & 10 \\
\hline \multirow{3}{*}{30} & 2 & 5571.92 & 1 & $0 \%$ & 8556.85 & 57.15 & 100.0 & 100.0 & 26 \\
\hline & 10 & 5571.92 & 1 & $0 \%$ & 9458.21 & 51.86 & 41.62 & 21.79 & 26 \\
\hline & 20 & 5571.92 & 1 & $0 \%$ & 9417.31 & 51.65 & 28.11 & 10.79 & 26 \\
\hline \multicolumn{10}{|c|}{ TR Model } \\
\hline \multirow{2}{*}{10} & 2 & 5571.92 & 1 & $0 \%$ & 6040.58 & 48.5 & 54.01 & 54.01 & 1 \\
\hline & 5 & 5571.92 & 1 & $0 \%$ & 6130.28 & 52.3 & 26.00 & 15.16 & 1 \\
\hline \multirow{2}{*}{20} & 2 & 5571.92 & 1 & $0 \%$ & 8420.46 & 46.35 & 68.38 & 68.38 & 2 \\
\hline & 10 & 5571.92 & 1 & $0 \%$ & 8465.03 & 51.19 & 31.17 & 14.82 & 2 \\
\hline \multirow{3}{*}{30} & 2 & 5571.92 & 1 & $0 \%$ & 8556.85 & 54.05 & 98.51 & 98.51 & 2 \\
\hline & 10 & 5571.92 & 1 & $0 \%$ & 10687.25 & 51.67 & 43.34 & 24.33 & 3 \\
\hline & 20 & 5571.92 & 1 & $0 \%$ & 10018.19 & 51.50 & 29.63 & 11.06 & 3 \\
\hline
\end{tabular}

\subsubsection{Varying $\mathcal{M}_{\alpha i}^{h k}$ for Fixed $\mathcal{P}_{\alpha i}^{h}$}

Once again, we consider the case of O-D Pair $(1,22)$. Table 4 compares the computational results for generating 10 dissimilar paths from $k=20,30,50$ and 80 candidate paths $\mathcal{M}_{0.999995,(1,22)}^{\text {Benzyl Chloride, }}$. From this table we conclude that a larger number of candidate paths yield a superior value of path dissimilarity. However, a larger number of candidate paths also result in a large average VaR value.

Another observation is that the generation of candidate paths is computationally expensive using the Removing Path Algorithm. Thus, there is a need to balance computational efficiency with solution quality. A small number of candidate paths allows a smaller number of VaR candidate paths but needs less computational effort; such a strategy but may exclude good dissimilar paths. In contrast, a larger number of candidate paths needs more computational effort and may result in a large VaR path set, but typically includes good dissimilar paths.

As shown in Table 4, the 10 out of 80 set generates solutions with larger minimal path dissimilarity (MiDi), and average path dissimilarity (AvDi). However, its solution requires more computational effort than the other data sets. Thus, there is a trade off between solution quality and efficiency. Comparing with TR model, we can see except for the 10 out of 20 set cases. VaR model generate solutions with paths with superior risk equities and smaller average VaRs. However, it is noted that 
Table 4: Computational Results for Dissimilar Hazmat Path-VaR Model Under $\alpha=0.999995$ vs. TR Model, O-D Pair $(1,22)$

\begin{tabular}{|c|c|c|c|c|c|c|c|c|c|}
\hline$|\mathcal{P}|$ & $|\mathcal{M}|$ & MiVaR & $\begin{array}{l}\text { Rank } \\
\text { in } \mathcal{M}\end{array}$ & $\begin{array}{c}\text { Gap } \\
\text { (from Opt) }\end{array}$ & $\mathrm{AvVaR}$ & AvLen & $\mathrm{AvDi}$ & $\mathrm{MiDi}$ & $\begin{array}{l}\text { Time } \\
(\mathrm{sec})\end{array}$ \\
\hline \multicolumn{10}{|c|}{$V R_{0.999995}$ Model } \\
\hline \multirow{4}{*}{10} & 20 & 5571.92 & 1 & $0 \%$ & 8303.45 & 51.35 & 32.46 & 15.16 & 10 \\
\hline & 30 & 5571.92 & 1 & $0 \%$ & 9458.21 & 51.86 & 41.62 & 21.79 & 26 \\
\hline & 50 & 5571.92 & 1 & $0 \%$ & 9512.77 & 54.41 & 42.73 & 24.33 & 101 \\
\hline & 80 & 5571.92 & 1 & $0 \%$ & 10203.28 & 55.41 & 46.14 & 28.53 & 396 \\
\hline \multicolumn{10}{|c|}{ TR Model } \\
\hline \multirow{4}{*}{10} & 20 & 5571.92 & 1 & $0 \%$ & 8465.03 & 51.19 & 31.17 & 14.82 & 2 \\
\hline & 30 & 5571.92 & 1 & $0 \%$ & 10687.25 & 51.67 & 43.34 & 24.33 & 3 \\
\hline & 50 & 5571.92 & 1 & $0 \%$ & 12088.80 & 53.61 & 51.01 & 30.28 & 4 \\
\hline & 80 & 5571.92 & 1 & $0 \%$ & 13244.77 & 54.93 & 53.08 & 31.30 & 7 \\
\hline
\end{tabular}

the TR model is more efficient than the VaR model, leading again to a tradeoff between solution quality and efficiency.

\subsubsection{Ten O-D Pair Observation}

Table 5 presents the 10 out of 30 dissimilar path solutions for ten different O-D pairs. In this computational experiment, we verify that, with rational size of candidate sets and dissimilar path sets, most O-D pairs can obtain a better dissimilar VaR path solution than the TR model, within acceptable computational time. The ten sets of dissimilar paths as well as the risk they bring to their neighborhoods are displayed in Table A.2 and compared with the optimal path of each O-D pair in Table A.1 in Appendix.

\subsection{Solution for Generalized Hazmat VaR Problem}

For the purpose of risk estimation, we use two types of hazmat, benzyl chloride and toluene, assuming a radius of spread of 1 mile and 3 miles, respectively. In order to compare the sensitivity of the size of the candidate set $\left|\mathcal{P}_{\alpha i}^{h}\right|$ to the result, we build two sets of $\mathcal{P}_{\alpha i}^{h}$, with 5 and 10 dissimilar paths individually for each type of hazmat between each O-D pair in our case. Both path sets come from the same 30 minimal VaR path set. In each of the geographical zones, we recount the road segments of each path and calculate the corresponding VaR values they bring to each zone. The data sets are formed by using various combinations of dissimilar path sets and types of hazmat shipped by the ten O-D pairs within the 7 and 28 geographical zones individually. Table A.1 displays the path VaR 
Table 5: Computational Results for Dissimilar VaR Hazmat Problem Under $\alpha=0.999995$, $\left|\mathcal{M}_{0.999995, i}^{\text {Benzyl Chloride, } 30}\right|=30,\left|\mathcal{P}_{0.999995,(1,22)}^{\text {Benzyl Chloride }}\right|=10$

\begin{tabular}{|c|c|c|c|c|c|c|c|c|}
\hline O-D Pair & MiVaR & Rank in $\mathcal{M}$ & Gap & $\mathrm{AvVaR}$ & MiLen & MiDi & $\mathrm{AvDi}$ & Total Time (sec) \\
\hline \multicolumn{9}{|c|}{$\mathrm{VaR}_{0.999995}$ Model } \\
\hline$(1,11)$ & 5571.92 & 1 & $0 \%$ & 8873.15 & 53.71 & 20.08 & 34.92 & 28 \\
\hline$(13,12)$ & 4908.01 & 1 & $0 \%$ & 5064.01 & & 23.88 & 38.90 & 20 \\
\hline$(1,22)$ & 5571.92 & 1 & $0 \%$ & 9458.21 & 51.95 & 24.29 & 41.62 & 29 \\
\hline$(1,12)$ & 4908.01 & 1 & $0 \%$ & 5052.45 & 70.70 & 18.89 & 32.57 & 30 \\
\hline$(2,35)$ & 8362.38 & 1 & $0 \%$ & 26945.56 & 26.94 & 35.85 & 47.26 & 23 \\
\hline$(3,11)$ & 5571.92 & 1 & $0 \%$ & 12622.28 & 34.64 & 27.04 & 38.83 & 19 \\
\hline$(2,11)$ & 8362.38 & 1 & $0 \%$ & 12439.07 & 38.54 & 27.20 & 35.06 & 26 \\
\hline$(75,21)$ & 11541.78 & 1 & $0 \%$ & 12825.99 & 46.07 & 23.63 & 28.52 & 22 \\
\hline$(1,10)$ & 5571.92 & 1 & $0 \%$ & 9150.38 & 50.35 & 22.26 & 38.17 & 26 \\
\hline$(76,28)$ & 11541.78 & 1 & $0 \%$ & 12445.14 & 35.41 & 20.20 & 30.89 & 17 \\
\hline \multicolumn{9}{|l|}{ TR Model } \\
\hline$(1,11)$ & 5571.92 & 1 & $0 \%$ & 8303.45 & 53.76 & 21.13 & 33.41 & 3 \\
\hline$(13,12)$ & 4908.01 & 1 & $0 \%$ & 5507.30 & & 24.89 & 38.28 & 3 \\
\hline$(1,22)$ & 5571.92 & 1 & $0 \%$ & 10687.35 & 51.67 & 24.95 & 43.34 & 3 \\
\hline$(1,12)$ & 4908.01 & 1 & $0 \%$ & 5087.77 & 70.76 & 20.83 & 32.85 & 4 \\
\hline$(2,35)$ & 8362.38 & 1 & $0 \%$ & 27190.19 & 25.51 & 35.43 & 50.46 & 2 \\
\hline$(3,11)$ & 5571.92 & 1 & $0 \%$ & 15079.71 & 34.12 & 29.59 & 40.51 & 2 \\
\hline$(2,11)$ & 8362.38 & 1 & $0 \%$ & 16138.89 & 37.72 & 27.26 & 36.57 & 2 \\
\hline$(75,21)$ & 11541.78 & 1 & $0 \%$ & 13621.91 & 44.52 & 14.92 & 25.40 & 1 \\
\hline$(1,10)$ & 5571.92 & 1 & $0 \%$ & 11334.17 & 50.43 & 23.78 & 43.73 & 3 \\
\hline$(76,28)$ & 11541.78 & 1 & $0 \%$ & 13466.79 & 33.46 & 13.70 & 30.40 & 1 \\
\hline
\end{tabular}

values and zone VaR values of each candidate path of the $10 \mathrm{O}-\mathrm{D}$ pairs.

\subsubsection{Impact of Size of $\mathcal{P}_{\alpha i}^{h}$}

Table 6 compares the results generated by 5 and 10 sets of dissimilar candidate paths $\mathcal{P}_{\alpha i}^{h}$ individually, under different risk threshold $\mu$. In the VaR model, by allowing more paths in the candidate path set, the total VaR we can get for the whole network can be lowered. That is, small dissimilar path sets may exclude low risk paths from being selectable. We note that the TR model gives us an opposite trend.

\subsubsection{Impact of Zone Segmentation}

In order to compare the impact of zone segmentation to our result, we segmented the network into 7 zones and 28 zones individually. Table 6 shows us in our case study, that with more segmented zones, more evenly the risks are spread, which in turn results in more mitigated VaRs. Comparing with TR model, we found that zone segmentation has a more obvious effect on the VaR model 
Table 6: Computational Results for Generalized VaR Hazmat Problem Under $\alpha=0.999995$

\begin{tabular}{|c|c|c|c|c|c|}
\hline \multicolumn{3}{|c|}{28 Zones } & \multicolumn{3}{|c|}{7 Zones } \\
\hline $\begin{array}{l}\text { Equity } \\
\mu\end{array}$ & $\begin{array}{l}\text { Total VaR } \\
\left(\left|\mathcal{P}_{\alpha i}^{h}\right|=5\right)\end{array}$ & $\begin{array}{l}\text { Total VaR } \\
\left(\left|\mathcal{P}_{\alpha i}^{h}\right|=10\right)\end{array}$ & $\begin{array}{l}\text { Equity } \\
\mu\end{array}$ & $\begin{array}{l}\text { Total VaR } \\
\left(\left|\mathcal{P}_{\alpha i}^{h}\right|=5\right)\end{array}$ & $\begin{array}{l}\text { Total VaR } \\
\left(\left|\mathcal{P}_{\alpha i}^{h}\right|=10\right)\end{array}$ \\
\hline \multicolumn{6}{|c|}{ VaR $_{0.999995}$ Model } \\
\hline 1000 & 1346949.01 & 1324372.46 & 5000 & 2235933.45 & 1829593.51 \\
\hline 2000 & 1357326.77 & 1335766.61 & 10000 & 2235933.45 & 1842297.19 \\
\hline 4000 & 1357326.77 & 1340338.38 & 20000 & 2233121.49 & 1801577.07 \\
\hline 6000 & 1357326.77 & 1340338.38 & 30000 & 2235933.45 & 1829593.51 \\
\hline 8000 & 1346949.01 & 1324372.46 & 40000 & 2231401.24 & 1829593.51 \\
\hline 10000 & 1346949.01 & 1309567.68 & 50000 & 2207917.01 & 1842297.19 \\
\hline \multicolumn{6}{|c|}{ TR Model } \\
\hline 5 & 1216402.82 & 1513689.06 & 1 & 1337839.94 & 1352513.61 \\
\hline 10 & 1065680.67 & 1065680.67 & 2 & 1343190.37 & 1366004.01 \\
\hline 20 & 1065680.67 & 1065680.67 & 4 & 1274551.67 & 1349843.11 \\
\hline 30 & 1065680.67 & 1065680.67 & 6 & 1267614.70 & 1243295.32 \\
\hline 40 & 1065680.67 & 1065680.67 & 8 & 1185142.93 & 1161014.08 \\
\hline 50 & 1065680.67 & 1065680.67 & 10 & 1065680.67 & 1065680.67 \\
\hline
\end{tabular}

solution.

\subsubsection{Impact of Risk Equity Constraint $\mu$}

From Table 6, we have another important observation regarding the risk equity constraints. The risk equity constraint has a more linear effect on the TR model than on the VaR model. For the TR model, a loose $\mu$ yields small global risk but will most likely lead to large risk differences between different geographical zones. By contrast, picking a tight value for $\mu$ yields a good risk dispersion solution, but may result in infeasibility problems and may not permit the selection of low risk paths. However, in the VaR model, the risk equity constraint effect has no such obvious effect on the total VaR value.

Assume that we have 56 shipments to be shipped among 10 O-D pairs, carrying two different types of hazmats. After the candidate path selection procedure, we chose 5 dissimilar candidate paths for each shipment. The $10 \mathrm{O}-\mathrm{D}$ pairs and 5 candidate paths are presented in Table A.2 in Appendix. The problem we face is to select the best path for each shipment so that the total VaR is minimal. Table 7 displays the optimal allocation of trips on each candidate path under different $\mu$ thresholds. For example, the optimal solution places 5 shipments of benzyl chloride in Candidate Path 1 for O-D Pair 1 in the 28 Zone case. We notice that with stricter $\mu$, more trips are pushed to high risk paths in order to obtain better risk equity. With $\mu=5000$, more trips are assigned to the 
minimal VaR paths of the 10 O-D pairs. Table 8 and Table 9 give the cumulative VaR values in each geographical zone and the VaR difference between each zone pair. These two tables further demonstrate that the tight threshold $(\mu=1000)$ generates solutions with risk dispersed more evenly than a looser threshold $(\mu=5000)$. That means, $\mu=1000$ gives much smaller VaR differences between any zone pair than $\mu=5000$. WE also note that the case of more zone segments (28 Zones) is more sensitive to the risk equity constraint than having less zone segments(7 Zones).

\subsubsection{Computational Analysis}

Given the candidate path sets, with path VaR values and zone VaR values of each path preprocessed, CPLEX finds a solution quickly. On the average, it takes no more than 1 second to solve the problem. However, as we discussed in Section 5, CPLEX is not a good solver for the VaR hazmat problem for large-scale networks, even with the given candidate path sets and all other information preprocessed. The Lagrangian relaxation heuristic provides an effective method to solve large-scale problems. Table 10 displays the computational results of Lagrangian relaxation and its gap from the Linear Programming lower bound, as well as a comparison with the CPLEX computational effort on the original integer programming problem. It demonstrates the efficiency of Lagrangian relaxation as a solution method for this problem.

CPLEX is widely used for solving MIP problems and recently CPLEX 12.4 has been successful at many types of MIPs. However, as the problem size increases for an MIP, CPLEX usually sees an exponential growth in solution time, rendering it unsuitable for large problem instances. Methods like parameter tuning and jump starting CPLEX with a good solution simply delay the onset of the exponential growth in solution time with problem size, but do not prevent this from happening. Therefore, a Lagrangian heuristic is still very much a viable solution method for large problems, especially when the Lagrangian sub-problems are easy to solve as in the problem of this paper.

A potential problem of the Lagrangian relaxation approach lies in the fact that relaxing constraint set (5) and solving the relaxed problem tends to result in all the trips with the same O-D pair carrying the same type of hazmat on an identical route. It is clear that a solution with exactly same routes is undesirable for a multi-trip problem, as it exposes population near a path multiple times and does not expose other areas to any risk. But with good design of the dissimilar candidate path sets for each O-D pair carrying each type of hazmat, we have dispersed the risk brought by 
Table 7: The Number of Shipments Assigned to Each O-D Pair and Each Path, Obtained by the Generalized VaR Hazmat Problem With 5 Candidate Path Sets for 10 O-D Pairs Under $\alpha=0.999995$ with 7 and 28 Zones Cases

\begin{tabular}{|c|c|c|c|c|c|c|c|c|c|c|c|c|c|}
\hline \multicolumn{14}{|c|}{$\mathrm{VaR}_{0.999995}$ Model } \\
\hline \multirow{3}{*}{ Hazmat Type } & \multirow{3}{*}{ O-D Pair } & \multicolumn{6}{|c|}{28 Zones } & \multicolumn{6}{|c|}{7 Zones } \\
\hline & & \multirow{2}{*}{$\begin{array}{c}\text { Equity } \\
\mu\end{array}$} & \multicolumn{5}{|c|}{ Candidate Path } & \multirow{2}{*}{$\begin{array}{c}\text { Equity } \\
\mu\end{array}$} & \multicolumn{5}{|c|}{ Candidate Path } \\
\hline & & & 1 & 2 & 3 & 4 & 5 & & 1 & 2 & 3 & 4 & 5 \\
\hline \multirow{10}{*}{ benzyl chloride } & 1 & \multirow{20}{*}{1000} & 5 & 0 & 0 & 0 & 0 & \multirow{20}{*}{5000} & 5 & 0 & 0 & 0 & 0 \\
\hline & 2 & & 0 & 0 & 0 & 2 & 0 & & 0 & 0 & 2 & 0 & 0 \\
\hline & 3 & & 3 & 0 & 0 & 0 & 0 & & 0 & 0 & 0 & 3 & 0 \\
\hline & 4 & & 0 & 0 & 0 & 2 & 0 & & 0 & 0 & 0 & 2 & 0 \\
\hline & 5 & & 0 & 0 & 0 & 0 & 4 & & 0 & 0 & 0 & 4 & 0 \\
\hline & 6 & & 0 & 0 & 0 & 0 & 3 & & 0 & 0 & 0 & 3 & 0 \\
\hline & 7 & & 0 & 0 & 5 & 0 & 0 & & 0 & 0 & 0 & 5 & 0 \\
\hline & 8 & & 0 & 0 & 0 & 1 & 0 & & 0 & 1 & 0 & 0 & 0 \\
\hline & 9 & & 2 & 0 & 0 & 0 & 0 & & 2 & 0 & 0 & 0 & 0 \\
\hline & 10 & & 0 & 0 & 0 & 0 & 1 & & 0 & 1 & 0 & 0 & 0 \\
\hline \multirow{11}{*}{ toluene } & 1 & & 0 & 0 & 0 & 0 & 5 & & 5 & 0 & 0 & 0 & 0 \\
\hline & 2 & & 0 & 0 & 0 & 2 & 0 & & 2 & 0 & 0 & 0 & 0 \\
\hline & 3 & & 0 & 0 & 2 & 0 & 0 & & 0 & 0 & 0 & 2 & 0 \\
\hline & 4 & & 3 & 0 & 0 & 0 & 0 & & 3 & 0 & 0 & 0 & 0 \\
\hline & 5 & & 0 & 0 & 1 & 0 & 0 & & 0 & 0 & 1 & 0 & 0 \\
\hline & 6 & & 0 & 0 & 0 & 0 & 4 & & 0 & 0 & 0 & 4 & 0 \\
\hline & 7 & & 5 & 0 & 0 & 0 & 0 & & 0 & 0 & 0 & 5 & 0 \\
\hline & 8 & & 0 & 0 & 1 & 0 & 0 & & 0 & 0 & 1 & 0 & 0 \\
\hline & 9 & & 0 & 0 & 2 & 0 & 0 & & 0 & 2 & 0 & 0 & 0 \\
\hline & \multirow[t]{2}{*}{10} & & 0 & 0 & 2 & 0 & 0 & & 0 & 0 & 0 & 0 & 2 \\
\hline & & \multicolumn{6}{|c|}{ Total VaR $=1346949.01$} & \multicolumn{6}{|c|}{ Total VaR $=2235933.45$} \\
\hline \multirow{10}{*}{ benzyl chloride } & 1 & \multirow{20}{*}{6000} & 5 & 0 & 0 & 0 & 0 & & 5 & 0 & 0 & 0 & 0 \\
\hline & 2 & & 2 & 0 & 0 & 0 & 0 & & 0 & 0 & 2 & 0 & 0 \\
\hline & 3 & & 3 & 0 & 0 & 0 & 0 & & 0 & 0 & 0 & 3 & 0 \\
\hline & 4 & & 2 & 0 & 0 & 0 & 0 & & 0 & 0 & 0 & 2 & 0 \\
\hline & 5 & & 0 & 0 & 0 & 0 & 4 & & 0 & 0 & 0 & 4 & 0 \\
\hline & 6 & & 0 & 0 & 0 & 0 & 3 & & 0 & 0 & 0 & 3 & 0 \\
\hline & 7 & & 0 & 0 & 5 & 0 & 0 & & 0 & 0 & 0 & 5 & 0 \\
\hline & 8 & & 0 & 0 & 1 & 0 & 0 & & 0 & 1 & 0 & 0 & 0 \\
\hline & 9 & & 2 & 0 & 0 & 0 & 0 & & 2 & 0 & 0 & 0 & 0 \\
\hline & 10 & & 0 & 0 & 1 & 0 & 0 & 50000 & 0 & 1 & 0 & 0 & 0 \\
\hline \multirow{11}{*}{ toluene } & 1 & & 0 & 0 & 0 & 0 & 5 & & 5 & 0 & 0 & 0 & 0 \\
\hline & 2 & & 2 & 0 & 0 & 0 & 0 & & 2 & 0 & 0 & 0 & 0 \\
\hline & 3 & & 0 & 0 & 2 & 0 & 0 & & 0 & 0 & 0 & 2 & 0 \\
\hline & 4 & & 3 & 0 & 0 & 0 & 0 & & 3 & 0 & 0 & 0 & 0 \\
\hline & 5 & & 0 & 0 & 1 & 0 & 0 & & 0 & 0 & 1 & 0 & 0 \\
\hline & 6 & & 0 & 0 & 0 & 0 & 4 & & 0 & 0 & 0 & 4 & 0 \\
\hline & 7 & & 0 & 0 & 5 & 0 & 0 & & 0 & 0 & 0 & 5 & 0 \\
\hline & 8 & & 0 & 0 & 1 & 0 & 0 & & 0 & 0 & 1 & 0 & 0 \\
\hline & 9 & & 0 & 0 & 2 & 0 & 0 & & 0 & 2 & 0 & 0 & 0 \\
\hline & 10 & & 0 & 0 & 2 & 0 & 0 & & 0 & 0 & 2 & 0 & 0 \\
\hline & & Total & $\mathrm{VaI}$ & $=$ & $135^{\prime}$ & ס & & Tota & $J$ & - & 20 & O & \\
\hline
\end{tabular}


Table 8: Zone VaR Comparison for Generalized VaR Hazmat Problem With 5 Candidate Path Sets for 10 O-D Pairs Under $\alpha=0.999995$

\begin{tabular}{|c|c|c|c|c|c|c|}
\hline \multirow{2}{*}{ Zone } & \multicolumn{2}{|c|}{ Equity $\mu=1000$} & \multicolumn{2}{|c|}{ Equity $\mu=6000$} & \multicolumn{2}{|c|}{ Equity $\mu=10000$} \\
\hline & benzyl chloride & toluene & benzyl chloride & toluene & benzyl chloride & toluene \\
\hline 1 & 0.00 & 83865.11 & 0.00 & 0.00 & 0.00 & 83865.11 \\
\hline 2 & 0.00 & 219744.51 & 0.00 & 0.00 & 0.00 & 219744.51 \\
\hline 3 & 51274.61 & 0.00 & 51274.61 & 98967.67 & 51274.61 & 0.00 \\
\hline 4 & 0.00 & 0.00 & 0.00 & 0.00 & 0.00 & 0.00 \\
\hline 5 & 0.00 & 0.00 & 0.00 & 0.00 & 0.00 & 0.00 \\
\hline 6 & 0.00 & 0.00 & 0.00 & 0.00 & 0.00 & 0.00 \\
\hline 7 & 0.00 & 0.00 & 0.00 & 0.00 & 0.00 & 0.00 \\
\hline 8 & 13011.91 & 150114.57 & 13011.91 & 150114.57 & 13011.91 & 150114.57 \\
\hline 9 & 0.00 & 0.00 & 0.00 & 0.00 & 0.00 & 0.00 \\
\hline 10 & 0.00 & 0.00 & 13217.52 & 0.00 & 13217.52 & 0.00 \\
\hline 11 & 0.00 & 0.00 & 0.00 & 0.00 & 0.00 & 0.00 \\
\hline 12 & 0.00 & 0.00 & 0.00 & 0.00 & 0.00 & 0.00 \\
\hline 13 & 0.00 & 0.00 & 0.00 & 0.00 & 0.00 & 0.00 \\
\hline 14 & 0.00 & 0.00 & 0.00 & 0.00 & 0.00 & 0.00 \\
\hline 15 & 0.00 & 0.00 & 0.00 & 0.00 & 0.00 & 0.00 \\
\hline 16 & 4849.90 & 32222.87 & 4849.90 & 32222.86 & 4849.90 & 32222.87 \\
\hline 17 & 0.00 & 0.00 & 0.00 & 0.00 & 0.00 & 0.00 \\
\hline 18 & 12287.42 & 22794.33 & 12287.42 & 0.00 & 12287.42 & 22794.33 \\
\hline 19 & 0.00 & 0.00 & 0.00 & 0.00 & 0.00 & 0.00 \\
\hline 20 & 0.00 & 0.00 & 0.00 & 79605.18 & 0.00 & 0.00 \\
\hline 21 & 0.00 & 0.00 & 0.00 & 0.00 & 0.00 & 0.00 \\
\hline 22 & 0.00 & 0.00 & 0.00 & 0.00 & 0.00 & 0.00 \\
\hline 23 & 0.00 & 0.00 & 0.00 & 40856.70 & 0.00 & 0.00 \\
\hline 24 & 0.00 & 0.00 & 0.00 & 0.00 & 0.00 & 0.00 \\
\hline 25 & 0.00 & 0.00 & 0.00 & 0.00 & 0.00 & 0.00 \\
\hline 26 & 0.00 & 0.00 & 0.00 & 0.00 & 0.00 & 0.00 \\
\hline 27 & 0.00 & 0.00 & 0.00 & 0.00 & 0.00 & 0.00 \\
\hline 28 & 0.00 & 0.00 & 0.00 & 0.00 & 0.00 & 0.00 \\
\hline
\end{tabular}

different candidate paths. Besides, a proper selection of the risk equity threshold $\mu$ can help direct the trips between the same O-D pair carrying the same type of hazmat onto different dissimilar candidate paths. In the model proposed by [17], they provide a heuristic solution procedure to avoid assigning all trips to the same optimal path by iteratively using the solution procedure of the single-trip problem. A similar approach can certainly be used in our situation.

\section{Concluding Remarks}

This paper aims to generate a generalized method for the multi-trip hazmat routing plan problem with a newly proposed VaR risk model and risk equity constraints. The VaR risk model measures 
Table 9: 7 Zone Pair VaR Difference for Generalized Hazmat VaR Problem With 5 Candidate Path Sets for 10 O-D Pairs Under $\alpha=0.999995$

\begin{tabular}{|c|c|c|c|c|c|c|c|c|}
\hline \multirow{2}{*}{$\begin{array}{c}\text { Equity } \\
\mu\end{array}$} & \multirow[b]{2}{*}{ Zone } & \multicolumn{7}{|c|}{ Zone } \\
\hline & & 1 & 2 & 3 & 4 & 5 & 6 & 7 \\
\hline \multirow{7}{*}{5000} & 1 & 0 & 107728.43 & 139799.65 & 53575.14 & 91892.60 & 137545.65 & 0 \\
\hline & 2 & 107728.43 & 0 & 32071.22 & 54153.29 & 15835.83 & 29817.22 & 107728.43 \\
\hline & 3 & 139799.65 & 32071.22 & 0 & 86224.51 & 47907.05 & 2254.00 & 139799.65 \\
\hline & 4 & 53575.14 & 54153.29 & 86224.51 & 0 & 38317.46 & 83970.51 & 53575.14 \\
\hline & 5 & 91892.60 & 15835.83 & 47907.05 & 38317.46 & 0 & 45653.05 & 91892.60 \\
\hline & 6 & 137545.65 & 29817.22 & 2254.00 & 83970.51 & 45653.05 & 0 & 137545.65 \\
\hline & 7 & 0 & 195806.08 & 139799.65 & 53575.14 & 91892.60 & 137545.65 & 0 \\
\hline \multirow{7}{*}{50000} & 1 & 0 & 13217.52 & 139799.65 & 53575.14 & 91892.60 & 137545.65 & 0 \\
\hline & 2 & 13217.52 & 0 & 126582.13 & 40357.62 & 78675.08 & 124328.13 & 13217.52 \\
\hline & 3 & 139799.65 & 126582.13 & 0 & 86224.51 & 47907.05 & 2254.00 & 139799.65 \\
\hline & 4 & 53575.14 & 40357.62 & 86224.51 & 0 & 38317.46 & 83970.51 & 53575.14 \\
\hline & 5 & 91892.60 & 78675.08 & 47907.05 & 38317.46 & 0 & 45653.05 & 91892.60 \\
\hline & 6 & 137545.65 & 124328.13 & 2254.00 & 83970.51 & 45653.05 & 0 & 137545.65 \\
\hline & 7 & 0 & 13217.52 & 139799.65 & 53575.14 & 91892.60 & 137545.65 & 0 \\
\hline
\end{tabular}

risks brought by hazmat transport under uncertainties. Due to the unique characteristics of hazmat transport, none of finance VaR methodologies can be applied. Effective solution methods are developed in this paper include finding the $k$ best VaR paths, $\mathcal{P}_{\alpha}$-dispersion, and Lagrangian relaxation. Different cases are discussed for both small-sized and large-sized road network, with corresponding solution methods provided. This generalized hazmat VaR problem represents the general hazmat routing problems in real life, as it allows multiple O-D pairs, multiple hazmat types, and multiple trips. It also allows equity of risk consideration. The equity constraint is usually the most important in the context of hazmat routing but can be incorporated within the context of many

Table 10: Computational Results Comparison Before vs. After Lagrangian Relaxation

\begin{tabular}{|c|c|c|c|c|c|c|c|}
\hline \multirow{2}{*}{$\begin{array}{c}\# \text { O-D } \\
\text { Pairs }\end{array}$} & \multicolumn{3}{|c|}{ Objective Function Value } & \multirow{2}{*}{$\begin{array}{r}\text { Gap } \\
(\mathrm{UB} \text { vs. LB) }\end{array}$} & \multicolumn{3}{|c|}{ Total Time } \\
\hline & IP & $\mathrm{LB}$ & UB & & IP & $\mathrm{LB}$ & UB \\
\hline 100 & 10069.80 & 10069.48 & 10084.92 & $0.15 \%$ & $3 \mathrm{~min} 37 \mathrm{sec}$ & $2 \mathrm{~min} 42 \mathrm{sec}$ & $1 \mathrm{~min} 4 \mathrm{sec}$ \\
\hline 200 & 19955.34 & 19954.87 & 19955.34 & $0.00 \%$ & $15 \mathrm{~min} 32 \mathrm{sec}$ & $10 \mathrm{~min} 40 \mathrm{sec}$ & $1 \mathrm{~min} 37 \mathrm{sec}$ \\
\hline 400 & - & 39568.62 & 39813.12 & $0.62 \%$ & - & $38 \mathrm{~min} 16 \mathrm{sec}$ & $2 \mathrm{~min} 9 \mathrm{sec}$ \\
\hline 800 & - & 80035.03 & 80481.75 & $0.56 \%$ & - & $153 \mathrm{~min} 39 \mathrm{sec}$ & $3 \mathrm{~min} 52 \mathrm{sec}$ \\
\hline 1000 & - & 99444.86 & 99914.45 & $0.47 \%$ & - & $240 \mathrm{~min} 40 \mathrm{sec}$ & $4 \mathrm{~min} 34 \mathrm{sec}$ \\
\hline \multicolumn{8}{|c|}{$\begin{array}{l}\text { Note: } \\
\text { IP: Integer Programming Solution by CPLEX } \\
\text { LB: Lower Bound by Linear Programming Relaxa } \\
\text { UB: Upper Bound by Lagrangian Relaxation } \\
\text { '-': too slow to finish within allowable time } \\
\text { \# of Paths for Each O-D Pair } \times \# \text { of Hazmat Tyl }\end{array}$} \\
\hline
\end{tabular}


scheduling/assignment problems. The main focus of our paper is on generic equity modeling for the hazmat transport problem, independent of the kind of risk measurement method applied. Clearly, other practical constraints are needed as are more advanced heuristics like column generation. The exploration of these avenues is suggested for future research.

There are several other directions for future research. One possibility is to relax the underlying assumption in this paper that all link attributes including accident probability and consequence are known. Although most research results reported in the literature rely on the assumption of known attributes, it is obvious that we need routing methods that are robust to inaccuracy of data and less sensitive to changes in link attributes. One possible strategy is to employ robust optimization methods, such as the method of Kwon et al. [27]. Regarding the methodology this paper applied, we narrow down the search space to a small set of candidate paths by $k$ best VaR paths and $\mathcal{P}_{\alpha}$-dispersion, in order to raise solution efficiency. It needs very careful of search space selection, and it may exclude some real good solutions. One potential extension can be heuristics like column generation for iteratively enhancing the candidate set of paths. That is, we start from a small set of candidate paths and add one path in each iteration when the outcome is not satisfactory. Besides, as we mentioned in literature review and Section 4, the $p$-dispersion method is more effective in generating a set of dissimilar paths. However, the solution generated by the $p$-dispersion method does not necessarily include the least risk path. It may be inappropriate for any decision maker to accept a set of routes not including the best path. A potential improvement can be placing restrictions on the size of candidate path sets and dissimilar path sets, or by enforcing constraints on the risk threshold. Other directions of future research include application extensions, like allowing curfews and road bans to regulate hazmat movement. Our current research assumes that there are no time-varying impacts.

\section{Acknowledgement}

This research was partially supported by the National Science Foundation under Grant Number CMMI-1068585. Any opinions, findings, and conclusions or recommendations expressed in this paper are those of the authors and do not necessarily reflect the views of the National Science Foundation. 


\section{References}

[1] M. Abkowitz and P.D. Cheng. Developing a Risk/Cost Framework for Routing Truck Movements of Hazardous Materials. Accident Analysis and Prevention, 20(1):39 - 51, 1988.

[2] M. Abkowitz, A. Eiger, and S. Srinivasan. Estimating the Release Rates and Costs of Transporting Hazardous Waste. Transportation Research Record, 977:22 - 30, 1984.

[3] V. Akgün, E. Erkut, and R. Batta. On Finding Dissimilar Paths. European Journal of Operational Research, 121(2):232 - 246, 2000.

[4] E. Alp. Risk-based Transportation Planning Practice: Overall Methodology and a Case Example. INFOR: Information Systems and Operations Research, 33(1):4 - 19, 1995.

[5] R. Batta and S.S. Chiu. Optimal Obnoxious Paths on a Network: Transportation of Hazardous Materials. Operations Research, 36(1):84 - 92, 1988.

[6] M.G.H. Bell. Mixed Routing Strategies for Hazardous Materials: Decision-making under Complete Uncertainty. International Journal of Sustainable Transportation, 1(2):133 - 142, 2007.

[7] L. Bianco, M. Caramia, and S. Giordani. A Bilevel Flow Model for Hazmat Transportation Network Design. Transportation Research Part C: Emerging Technologies, 17(2):175 - 196, 2009.

[8] P. Carotenuto, S. Giordani, and S. Ricciardelli. Finding Minimum and Equitable Risk Routes for Hazmat Shipments. Computers \& Operations Research, 34(5):1304 - 1327, 2007.

[9] J. Current and S. Ratick. A Model to Assess Risk, Equity and Efficiency in Facility Location and Transportation of Hazardous Materials. Location Science, 3(3):187 - 201, 1995.

[10] P. Dell'Olmo, M. Gentili, and A. Scozzari. On Finding Dissimilar Pareto-Optimal Paths. European Journal of Operational Research, 162(1):70 - 82, 2005.

[11] A. Duarte and R. Marti. Tabu Search and GRASP for the Maximum Diversity Problem. European Journal of Operational Research, 178(1):71 - 84, 2007. 
[12] D. Eppstein. Finding the $k$ Shortest Paths. SIAM Journal on Computing, 28(2):652 - 673, 1998.

[13] E. Erkut. The Discrete p-Dispersion Problem. European Journal of Operational Research, 46 (1):48 - 60, May 1990.

[14] E. Erkut and A. Ingolfsson. Catastrophe Avoidance Models for Hazardous Materials Route Planning. Transportation Science, 34(2):165 - 179, 2000.

[15] E. Erkut, Y. Ülküsal, and O. Yenicerioğlu. A Comparison of p-Dispersion Heuristics. Computers $\&$ Operations Research, 21(10):1103 - 1113, 1994.

[16] R. Gopalan, R. Batta, and M.H. Karwan. The Equity Constrained Shortest Path Problem. Computers $\&$ Operations Research, 17(3):297 - 307, 1990a.

[17] R. Gopalan, K.S. Kolluri, R. Batta, and M.H. Karwan. Modeling Equity of Risk in the Transportation of Hazardous Materials. Operations Research, 38(6):961 - 973, 1990b.

[18] D.W. Harwood, J.G. Viner, and E.R. Russell. Procedure for Developing Truck Accident and Release Rates for Hazmat Routing. Journal of Transportation Engineering, 119(1):189 -199, 1993.

[19] M. Held, P. Wolfe, and H.P. Crowder. Validation of Subgradient Optimization. Mathematical Programming, 6:62 - 88, 1974.

[20] H. Jin and R. Batta. Objectives Derived from Viewing Hazmat Shipments as a Sequence of Independent Bernoulli Trials. Transportation Science, 31(3):252 - 261, 1997.

[21] P.E. Johnson, D.S. Joy, D.B. Clarke, and J.M. Jacobi. HIGHWAY 3.01, An Enhanced Highway Routing Model: Program, Description, Methodology, and Revised User's Manual. Technial Report, Oak Ridge National Laboratory, 19(4):315 - 329, 1992.

[22] Y. Kang, R. Batta, and C. Kwon. Value-at-Risk Model for Hazardous Material Transportation. Annals of Operations Research, accepted, 2013.

[23] B.Y. Kara and V. Verter. Designing a Road Network for Hazardous Materials Transportation. Transportation Science, 38(2):188 - 196, 2004. 
[24] R.L. Keeney. Equity and Public Risk. Operations Rearch, 28(3-Part-I):527 - 534, 1980.

[25] M. Kuby, Z.Y. Xu, and X.D. Xie. A Minimax Method for Finding the k Best "Differentiated" Paths. Geographical Analysis, 29(4):298 - 313, October 1997.

[26] M.J. Kuby. Programming Models For Facility Dispersion: The p-Dispersion And Maximum Dispersion Problems. Geographical Analysis, 19(4):315 - 329, 1987.

[27] Changhyun Kwon, Taehan Lee, and Paul Berglund. Robust shortest path problems with two uncertain multiplicative cost coefficients. Naval Research Logistics, 60(5):375-394, 2013.

[28] L. Lindner-Dutton, R. Batta, and M.H. Karwan. Equitable Sequencing of a Given Set of Hazardous Materials Shipments. Transportation Science, 25(2):124 - 137, 1991.

[29] K. Lombard and R.L. Church. The Gateway Shortest Path Problem: Generating Alternative Routes For A Corridor Location Problem. Geographical Systems, 1:25 - 45, 1993.

[30] P. Marcotte, A. Mercier, G. Savard, and V. Verter. Toll Policies for Mitigating Hazardous Materials Transport Risk. Transportation Science, 43(2):228 - 243, 2009.

[31] R. Martí, J.L.G. Velarde, and A. Duarte. Heuristics for the Bi-Objective Path Dissimilarity Problem. Computers \& Operations Research, 36(11):2905 - 2912, 2009.

[32] E.Q.V. Martins. An Algorithm For Ranking Paths That May Contain Cycles. European Journal of Operational Research, 18(1):123 - 130, October 1984.

[33] M.H. Patel and A.J. Horowitz. Optimal Routing of Hazardous Materials. Considering Risk of Spill. Transportation Research Part A, 28(2):119 - 132, 1994.

[34] M.G.C. Resende and C.C. Ribeiro. Greedy Randomized Adaptive Search Procedures, 2003.

[35] C. ReVelle, J. Cohon, and D. Shobrys. Simultaneous Siting and Routing in the Disposal of Hazardous Wastes. Transportation Science, 25(2):138 - 145, 1991.

[36] F.F. Saccomanno and A.Y.W. Chan. Economic Evaluation of Routing Strategies for. Hazardous Road Shipments. Transportation Research Record, 1020:12 - 18, 1985. 
[37] R.A. Sivakumar, R. Batta, and M.H. Karwan. Establishing Credible Risk Criteria for Transporting Extremely Dangerous Hazardous Materials. In F.F. Saccomanno and K. Cassidy, editors, Transportation of Dangerous Goods: Assessing the Risks, pages 335 - 342. Institute for Risk Research, University of Waterloo, Canada, 1993.

[38] K. Thyagarajan, R. Batta, M.H. Karwan, and R.J. Szczerba. Planning Dissimilar Paths for Military Units. Military Operations Research, 10(1):25 - 42, 2005.

[39] U.S. Department of Transportation. Hazardous Materials Safety: Hazardous Materials Information System. Technical report, 2010. URL http://www.phmsa.dot.gov/staticfiles/PHMSA/DownloadableFiles/Files/tenyr.pdf.

[40] V. Verter and B.Y. Kara. A Path-Based Approach for Hazmat Transport Network Design. Management Science, 54(1):29 - 40, 2008.

[41] Jin Y. Yen. Finding the K Shortest Loopless Paths in a Network. Management Science, 17(11): $712-716,1971$.

[42] J. Zhang, J. Hodgson, and E. Erkut. Using GIS to Assess the Risks of Hazardous Materials Transport in Networks. European Journal of Operational Research, 121(2):316 - 329, 2000. 


\section{Appendix}

This appendix, which is provided for the reviewers' convenience, may be provided as an online supplementary document.

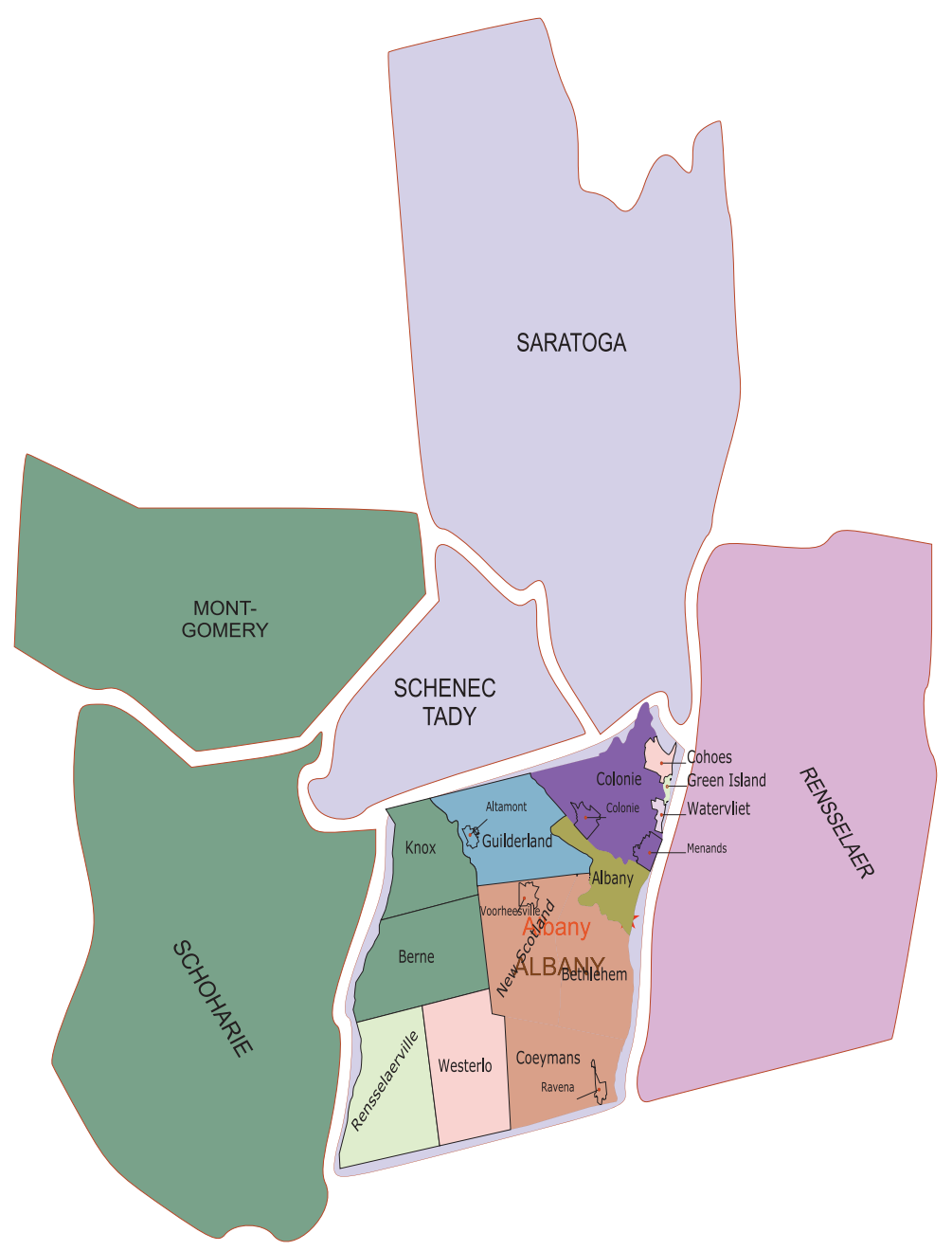

Figure A.1: Albany Area Township 
Table A.1: Computational Results for Single-Trip Single-Path VaR Hazmat Problem Under $\alpha=$ 0.999995

\begin{tabular}{rll}
\hline O-D Pair & Optimal Path & VaR value \\
\hline$(1,11)$ & {$[1,70,45,13,14,15,55,56,60,61,16,17,18,19,20,21,10,11]$} & 28204.81 \\
$(13,12)$ & {$[13,81,72,73,69,66,67,68,41,29,30,12]$} & 19557.14 \\
$(1,22)$ & {$[1,70,45,13,14,15,55,56,60,61,16,17,18,19,20,21,10,22]$} & 28204.81 \\
$(1,12)$ & {$[1,70,45,13,81,72,73,69,66,67,68,41,29,30,12]$} & 19557.14 \\
$(2,35)$ & {$[2,3,58,59,60,61,16,17,18,19,20,35]$} & 40329.18 \\
$(3,11)$ & {$[3,15,55,56,60,61,16,17,18,19,20,21,10,11]$} & 28204.81 \\
$(2,11)$ & {$[2,3,58,59,60,61,16,17,18,19,20,21,10,11]$} & 40329.18 \\
$(75,21)$ & {$[75,76,77,79,23,24,25,33,24,25,33,39,86,87,9,84,21]$} & 47121.44 \\
$(1,10)$ & {$[1,70,45,13,14,15,55,56,60,61,16,17,18,18,20,21,10]$} & 28294.81 \\
$(76,28)$ & {$[76,77,79,23,24,25,33,34,7,19,36,28]$} & 47255.46 \\
\hline
\end{tabular}


Table A.2: Computational Results for Single-Trip Multiple-Path Hazmat VaR Problem Under $\alpha=0.999995$

\begin{tabular}{|c|c|c|c|c|c|c|c|c|c|}
\hline \multirow{2}{*}{$\begin{array}{l}\text { O-D } \\
\text { Pair }\end{array}$} & \multirow[t]{2}{*}{ Dissimilar Path } & \multirow[t]{2}{*}{ Path VaR } & \multicolumn{7}{|c|}{ Zone VaR } \\
\hline & & & $Z_{1}$ & $Z_{2}$ & $Z_{3}$ & $Z_{4}$ & $Z_{5}$ & $Z_{6}$ & $Z_{7}$ \\
\hline \multirow[t]{5}{*}{$(1,11)$} & {$[1,70,45,13,14,15,55,56,60,61,16,17,18,19,20,21,10,11]$} & 28204.81 & 0 & 0 & 3743.38 & 0 & 0 & 1301.19 & 0 \\
\hline & {$[1,2,3,58,59,60,61,16,17,18,19,20,84,85,22,11]$} & 41932.56 & 0 & 0 & 0 & 0 & 11268.99 & 6259.98 & 0 \\
\hline & {$[1,70,45,71,58,57,56,60,61,16,17,18,19,20,84,21,10,22,11]$} & 32081.30 & 0 & 0 & 2768.16 & 0 & 0 & 5691.23 & 0 \\
\hline & {$[1,2,3,14,15,55,56,60,61,16,17,18,19,20,21,10,22,11]$} & 41932.56 & 0 & 0 & 3743.38 & 0 & 11268.99 & 2832.15 & 0 \\
\hline & {$[1,70,45,13,81,14,15,55,56,60,61,16,17,18,19,20,84,85,22,11]$} & 32245.04 & 0 & 0 & 3743.38 & 0 & 0 & 1679.30 & 0 \\
\hline \multirow[t]{5}{*}{$(13,12)$} & {$[13,81,72,73,69,66,67,68,41,29,30,12]$} & 19557.14 & 0 & 0 & 0 & 1212.48 & 0 & 0 & 0 \\
\hline & {$[13,14,15,55,56,60,61,16,17,18,19,20,21,10,11,12]$} & 28204.81 & 0 & 0 & 4109.09 & 5338.07 & 0 & 1266.82 & 0 \\
\hline & {$[13,14,15,55,56,60,61,62,63,64,65,54,66,67,68,41,29,30,12]$} & 26010.57 & 0 & 0 & 0 & 5338.07 & 3071.86 & 0 & 0 \\
\hline & {$[13,81,14,15,55,63,52,53,54,66,67,68,41,29,30,12]$} & 21813.39 & 0 & 0 & 3743.38 & 1212.48 & 0 & 1266.82 & 0 \\
\hline & {$[13,81,14,15,55,56,60,61,16,17,18,19,20,84,21,10,22,11,12]$} & 28204.81 & 0 & 0 & 0 & 1391.19 & 0 & 1679.30 & 0 \\
\hline \multirow[t]{5}{*}{$(1,22)$} & {$[1,70,45,13,14,15,55,56,60,61,16,17,18,19,20,21,10,22]$} & 28204.81 & 0 & 0 & 3743.38 & 0 & 0 & 1301.19 & 0 \\
\hline & {$[1,74,75,76,77,79,23,24,25,33,39,86,89,90,85,22]$} & 47121.43 & 0 & 0 & 0 & 0 & 10395.42 & 4735.00 & 0 \\
\hline & {$[1,2,3,58,59,60,61,16,17,18,19,20,84,85,22]$} & 41932.56 & 0 & 0 & 0 & 0 & 11268.99 & 6279.98 & 0 \\
\hline & {$[1,70,45,71,58,59,60,61,16,17,18,19,20,84,21,10,22]$} & 34021.98 & 0 & 0 & 0 & 0 & 0 & 5691.23 & 0 \\
\hline & {$[1,2,3,15,55,56,60,61,16,17,18,19,20,21,10,22]$} & 41932.56 & 0 & 0 & 3743.38 & 0 & 11268.99 & 2608.66 & 0 \\
\hline \multirow[t]{5}{*}{$(1,12)$} & {$[1,70,45,13,81,72,73,69,66,67,68,41,29,30,12]$} & 19557.14 & 0 & 0 & 0 & 1212.48 & 0 & 0 & 0 \\
\hline & {$[1,70,45,71,58,57,56,60,61,62,63,64,65,54,66,67,68,41,29,30,12]$} & 28184.11 & 0 & 0 & 4109.09 & 5338.07 & 0 & 1301.19 & 0 \\
\hline & {$[1,70,45,13,14,15,55,56,62,63,52,53,54,66,67,68,41,29,30,12]$} & 21813.39 & 0 & 0 & 3743.38 & 1212.48 & 0 & 1301.19 & 0 \\
\hline & {$[1,70,45,13,81,14,15,55,63,64,65,54,66,67,68,41,29,30,12]$} & 19557.14 & 0 & 0 & 0 & 5338.07 & 3071.86 & 0 & 0 \\
\hline & {$[1,70,45,13,81,72,73,63,52,53,54,66,67,68,41,29,30,12]$} & 19557.14 & 0 & 0 & 0 & 1391.19 & 0 & 1679.30 & 0 \\
\hline \multirow[t]{4}{*}{$(2,35)$} & {$[2,3,58,59,60,61,16,17,18,19,20,35]$} & 40329.17 & 0 & 0 & 0 & 0 & 0 & 6259.98 & 0 \\
\hline & {$[2,44,43,42,25,26,34,7,8,20,35]$} & 161098.39 & 0 & 0 & 0 & 0 & 0 & 28901.87 & 0 \\
\hline & {$[2,3,4,5,6,7,8,20,35]$} & 145132.47 & 0 & 0 & 0 & 0 & 0 & 0 & 0 \\
\hline & {$[2,44,59,4,5,17,18,19,20,35]$} & 156873.21 & 0 & 0 & 0 & 0 & 0 & 0 & 0 \\
\hline \multicolumn{10}{|c|}{ Continued on next page } \\
\hline
\end{tabular}


Table A.2 - continued from previous page

\begin{tabular}{|c|c|c|c|c|c|c|c|c|c|}
\hline \multirow{2}{*}{$\begin{array}{l}\text { O-D } \\
\text { Pair }\end{array}$} & \multirow[t]{2}{*}{ Dissimilar Path } & \multirow[t]{2}{*}{ Path VaR } & \multicolumn{7}{|c|}{ Zone VaR } \\
\hline & & & $Z_{1}$ & $Z_{2}$ & $Z_{3}$ & $Z_{4}$ & $Z_{5}$ & $Z_{6}$ & $Z_{7}$ \\
\hline & {$[2,44,43,42,82,27,5,6,18,19,20,35]$} & 161098.39 & 0 & 0 & 3743.38 & 0 & 0 & 2832.15 & 0 \\
\hline \multirow[t]{5}{*}{$(3,11)$} & {$[3,15,55,56,60,61,16,17,18,19,20,21,10,11]$} & 28204.81 & 0 & 0 & 3743.38 & 0 & 0 & 0 & 0 \\
\hline & {$[3,58,59,4,5,17,18,19,20,84,85,22,11]$} & 122214.17 & 0 & 0 & 0 & 0 & 0 & 0 & 0 \\
\hline & {$[3,58,57,56,60,61,16,17,18,19,20,84,21,10,22,11]$} & 34021.97 & 0 & 0 & 2768.16 & 0 & 0 & 0 & 0 \\
\hline & {$[3,4,5,17,18,19,20,84,21,10,11]$} & 122214.17 & 0 & 0 & 0 & 0 & 0 & 0 & 0 \\
\hline & {$[3,14,15,55,56,60,61,16,17,18,19,20,84,85,22,11]$} & 32245.03 & 0 & 0 & 3743.38 & 0 & 0 & 0 & 0 \\
\hline \multirow[t]{5}{*}{$(2,11)$} & {$[2,3,58,59,60,61,16,17,18,19,20,21,10,11]$} & 40329.18 & 0 & 0 & 0 & 0 & 0 & 6259.98 & 0 \\
\hline & {$[2,3,4,5,17,18,19,20,84,85,22,11]$} & 122214.17 & 0 & 0 & 0 & 0 & 0 & 0 & 0 \\
\hline & {$[2,3,14,15,55,56,60,61,16,17,18,19,20,84,21,10,22,11]$} & 40329.18 & 0 & 0 & 3743.38 & 0 & 0 & 2832.15 & 0 \\
\hline & {$[2,3,58,59,4,5,17,18,19,20,21,10,22,11]$} & 122214.17 & 0 & 0 & 0 & 0 & 0 & 0 & 0 \\
\hline & {$[2,3,58,57,56,60,61,16,17,18,19,20,84,85,22,11]$} & 40329.18 & 0 & 0 & 2768.16 & 0 & 0 & 0 & 0 \\
\hline \multirow[t]{5}{*}{$(75,21)$} & {$[75,76,77,79,23,24,25,33,39,86,87,9,84,21]$} & 47121.44 & 0 & 0 & 0 & 0 & 0 & 4735.00 & 0 \\
\hline & {$[75,76,80,23,24,32,33,34,7,8,20,21]$} & 61921.22 & 0 & 6608.76 & 0 & 0 & 0 & 0 & 0 \\
\hline & {$[75,76,80,23,24,32,37,38,39,86,87,9,84,21]$} & 51582.29 & 0 & 0 & 0 & 0 & 0 & 0 & 0 \\
\hline & {$[75,76,77,79,23,24,32,33,34,7,19,20,21]$} & 61921.22 & 0 & 0 & 0 & 0 & 0 & 0 & 0 \\
\hline & {$[75,76,77,79,23,24,32,37,8,9,84,21]$} & 55937.04 & 0 & 0 & 0 & 0 & 0 & 4735.00 & 0 \\
\hline \multirow[t]{5}{*}{$(1,10)$} & {$[1,70,45,13,14,15,55,56,60,61,16,17,18,19,20,21,10]$} & 28204.81 & 0 & 0 & 3743.38 & 0 & 0 & 1301.19 & 0 \\
\hline & {$[1,74,75,76,77,79,23,24,25,33,39,86,87,9,10]$} & 47121.44 & 0 & 0 & 0 & 0 & 10395.42 & 4735.00 & 0 \\
\hline & {$[1,2,3,58,59,60,61,16,17,18,19,20,84,21,10]$} & 41932.55 & 0 & 0 & 0 & 0 & 11268.99 & 6259.98 & 0 \\
\hline & {$[1,70,45,71,58,57,56,60,61,16,17,18,19,20,84,21,10]$} & 32081.30 & 0 & 0 & 2768.16 & 0 & 0 & 5691.23 & 0 \\
\hline & {$[1,2,3,14,15,55,56,60,61,16,17,18,19,20,21,10]$} & 41932.56 & 0 & 0 & 3743.38 & 0 & 11268.99 & 2832.15 & 0 \\
\hline \multirow[t]{4}{*}{$(76,28)$} & {$[76,77,79,23,24,25,33,34,7,19,36,28]$} & 47255.46 & 0 & 0 & 0 & 0 & 0 & 4735.00 & 0 \\
\hline & {$[76,80,23,24,25,26,83,82,16,17,28]$} & 65590.51 & 0 & 6608.76 & 0 & 0 & 0 & 0 & 0 \\
\hline & {$[76,80,23,24,32,33,34,7,8,20,35,36,28]$} & 51582.29 & 0 & 0 & 0 & 0 & 0 & 0 & 0 \\
\hline & {$[76,77,79,23,24,25,26,83,82,27,5,17,28]$} & 62971.22 & 0 & 6608.76 & 0 & 0 & 0 & 4735.00 & 0 \\
\hline
\end{tabular}


Table A.2 - continued from previous page

\begin{tabular}{|c|c|c|c|c|c|c|c|c|c|}
\hline \multirow{2}{*}{$\begin{array}{l}\text { O-D } \\
\text { Pair }\end{array}$} & \multirow[t]{2}{*}{ Dissimilar Path } & \multirow[t]{2}{*}{ Path VaR } & \multicolumn{7}{|c|}{ Zone VaR } \\
\hline & & & $Z_{1}$ & $Z_{2}$ & $Z_{3}$ & $Z_{4}$ & $Z_{5}$ & $Z_{6}$ & $Z_{7}$ \\
\hline & {$[76,80,23,24,25,26,83,82,27,5,6,18,19,36,28]$} & 65590.51 & 0 & 0 & 0 & 0 & 0 & 4735.00 & 0 \\
\hline
\end{tabular}

